\title{
An approximation of the axially symmetric flow through a pipe-like domain with a moving part of a boundary
}

Jan Filo ${ }^{1 *}$ and Volker Pluschke 2

${ }^{*}$ Correspondence:

jan.filo@fmph.uniba.sk

${ }^{1}$ Department of Mathematical

Analysis and Numerical

Mathematics, Faculty of

Mathematics, Physics and

Informatics, Comenius University,

Mlynska dolina, Bratislava, 842 48,

Slovakia

Full list of author information is

available at the end of the article

\begin{abstract}
The purpose of this work is to study the existence of solutions for approximation of an unsteady fluid-structure interaction problem. We consider a perturbed Navier-Stokes equation in the cylindrical coordinate system assuming axially symmetric flow. A priori unknown part of the boundary (that interacts with the fluid) is governed with a linear equation of fifth order. We prove the existence of at least one weak solution as long as the boundary does not touch the axis of symmetry. An explicit expression for a class of divergence-free functions is given.
\end{abstract}

MSC: 35Q30; 35Q35; 35D05; 74F10; 76D03

Keywords: perturbed Navier-Stokes equations; artificial pressure; fluid-structure interaction; cylindrical coordinate system; Schauder fixed point theorem

\section{Introduction}

This paper is devoted to the perturbed Navier-Stokes equations in a cylindrical coordinate system assuming rotationally symmetric flow

$$
\left.\begin{array}{c}
\rho \frac{\partial v_{x}}{\partial t}+\rho v_{x} \frac{\partial v_{x}}{\partial x}+\rho v_{r} \frac{\partial v_{x}}{\partial r}+\frac{\rho}{2} v_{x}\left(\frac{\partial v_{x}}{\partial x}+\frac{\partial v_{r}}{\partial r}+\frac{1}{r} v_{r}\right) \\
=\mu\left\{2 \frac{\partial^{2} v_{x}}{\partial x^{2}}+\frac{1}{r} \frac{\partial}{\partial r}\left(r\left(\frac{\partial v_{x}}{\partial r}+\frac{\partial v_{r}}{\partial x}\right)\right)\right\}-\frac{\partial p}{\partial x}, \\
\rho \frac{\partial v_{r}}{\partial t}+\rho v_{x} \frac{\partial v_{r}}{\partial x}+\rho v_{r} \frac{\partial v_{r}}{\partial r}+\frac{\rho}{2} v_{r}\left(\frac{\partial v_{x}}{\partial x}+\frac{\partial v_{r}}{\partial r}+\frac{1}{r} v_{r}\right) \\
=\mu\left\{\frac{2}{r} \frac{\partial}{\partial r}\left(r \frac{\partial v_{r}}{\partial r}\right)+\frac{\partial}{\partial x}\left(\frac{\partial v_{r}}{\partial x}+\frac{\partial v_{x}}{\partial r}\right)-\frac{2}{r^{2}} v_{r}\right\}-\frac{\partial p}{\partial r}
\end{array}\right\}
$$

with an artificial equation for the pressure

$$
\varepsilon\left(\frac{\partial p}{\partial t}-\frac{\partial^{2} p}{\partial x^{2}}-\frac{1}{r} \frac{\partial}{\partial r}\left(r \frac{\partial p}{\partial r}\right)\right)+\frac{\partial v_{x}}{\partial x}+\frac{\partial v_{r}}{\partial r}+\frac{1}{r} v_{r}=0
$$

$(0<\varepsilon \ll 1)$ in an a priori unknown domain

$$
\Omega(h) \equiv\left\{(x, r, t):-L^{-}<x<L^{+}, 0<r<h(x, t), 0<t<T\right\},
$$

○2013 Filo and Pluschke; licensee Springer. This is an Open Access article distributed under the terms of the Creative Commons Attribution License (http://creativecommons.org/licenses/by/2.0), which permits unrestricted use, distribution, and reproduction in any medium, provided the original work is properly cited. 
where

$$
h(x, t)= \begin{cases}\chi(x) & \text { for }-L^{-} \leq x \leq 0 \text { and } L \leq x \leq L^{+}, \\ \chi(x)+\eta(x, t) & \text { for } 0 \leq x \leq L\end{cases}
$$

with the given

$$
\chi(x)= \begin{cases}R^{-}, & -L^{-} \leq x \leq 0, \\ \text { smooth and } \chi^{\prime} \leq 0 \text { on } & {\left[-L^{-}, L^{+}\right]} \\ R^{+}, & L \leq x \leq L^{+}\end{cases}
$$

and $\rho, \mu, L, L^{+}, T, R^{-}, R^{+}$are given positive constants, $L^{-} \geq 0, R^{+} \leq R^{-}$.

Unknown are the velocity field $\left(v_{x}, v_{r}\right)$, the pressure $p$ and the interface $\eta$ on $[0, L]$. We shall require from $\eta$ to satisfy the following conditions:

$$
\begin{aligned}
& \frac{\partial \eta}{\partial t}(x, t)=v_{r}(x, h(x, t), t), \\
& -E\left[\frac{\partial^{2} \eta}{\partial t^{2}}+e \frac{\partial^{5} \eta}{\partial t^{3} \partial x^{2}}+d \frac{\partial^{5} \eta}{\partial t \partial x^{4}}+f \frac{\partial^{4} \eta}{\partial x^{4}}-c \frac{\partial^{3} \eta}{\partial t \partial x^{2}}-a \frac{\partial^{2} \eta}{\partial x^{2}}+b \eta\right](x, t) \\
& \quad=h(x, t)\left[2 \mu \frac{\partial v_{r}}{\partial r}+\mu\left(\frac{\partial v_{r}}{\partial x}+\frac{\partial v_{x}}{\partial r}\right)\left(-\frac{\partial h}{\partial x}\right)-p+p_{w}\right](x, h(x, t), t)
\end{aligned}
$$

for $0<x<L, 0<t<T$,

$$
\eta(0, t)=\frac{\partial \eta}{\partial x}(0, t)=\eta(L, t)=\frac{\partial \eta}{\partial x}(L, t)=0
$$

for any $0<t<T$ and, for convenience, also

$$
\eta(x, 0)=0, \quad \frac{\partial \eta}{\partial t}(x, 0)=0, \quad 0 \leq x \leq L .
$$

Here $a, b, c, f$ are given nonnegative numbers and $d, e, E$ are supposed to be positive. Moreover, $p_{w}=p_{w}(x, t)$ is a given function. Of course, (1.1) is to be complemented with additional boundary and initial conditions. We shall consider

$$
v_{x}(x, h(x, t), t)=0
$$

for any $-L^{-}<x<L^{+}, 0<t<T$,

$$
v_{r}(x, h(x, t), t)=0
$$

for any $-L^{-} \leq x \leq 0$ and $L \leq x \leq L^{+}, 0<t<T$,

$$
\left(2 \mu \frac{\partial v_{x}}{\partial x}-p-p_{\text {out }}-\frac{\rho}{2}\left|v_{x}\right|^{2}\right)\left(L^{+}, r, t\right)=0, \quad v_{r}\left(L^{+}, r, t\right)=0
$$

for any $0<r<R^{+}, 0<t<T$,

$$
\left(2 \mu \frac{\partial v_{x}}{\partial x}-p+p_{\text {in }}-\frac{\rho}{2}\left|v_{x}\right|^{2}\right)\left(-L^{-}, r, t\right)=0, \quad v_{r}\left(-L^{-}, r, t\right)=0
$$


for any $0<r<R^{-}, 0<t<T$,

$$
\frac{\partial v_{x}}{\partial r}(x, 0, t)=0, \quad v_{r}(x, 0, t)=0
$$

for any $-L^{-}<x<L^{+}, 0<t<T$ and, finally,

$$
\left(v_{x}(x, r, 0), v_{r}(x, r, 0)\right)=(0,0)
$$

for any $-L^{-}<x<L^{+}, 0<r<h(x, 0)$. Here $p_{\text {in }}$ and $p_{\text {out }}$ are given functions of two variables $(r, t)$ for $0 \leq r \leq R^{-}$and $0 \leq r \leq R^{+}$, respectively, $0 \leq t \leq T$.

The aim of this paper is to extend the existence result of [1] concerning the twodimensional fluid-structure interaction problem to the radially symmetric case expressed in terms of the cylindrical coordinate system. Since we have not been able yet to handle this nonlinear problem modelling the flow of an incompressible viscous fluid in a cylinder with deformable wall, here nonlinear perturbed Navier-Stokes equations coupled with the artificial equation (1.2) and assuming $e>0$ in the equation of the wall (1.6) are studied. As it turns out, we have not been able to let

$$
\varepsilon \rightarrow 0
$$

up to now due to the lack of regularity in the time variable.

We can give a justification of this limit, but only for a linearised perturbed Navier-Stokes equations and by including the additional term

$$
-g \frac{\partial^{6} \eta}{\partial x^{6}} \quad(g>0)
$$

in (1.6). Let us illustrate some arguments for the presence of this term in (1.6). To prove our existence result, we shall transform $\Omega(h)$ for $h$ given by (1.4) on a fixed domain $D=$ $\left\{(x, y) \in \mathbb{R}^{2}:-L^{-}<x<L^{+}, 0<y<1\right\}$ by setting

$$
\left.\begin{array}{l}
\boldsymbol{u}(x, y, t)=\left(v_{x}(x, h(x, t) y, t), v_{r}(x, h(x, t) y, t)\right) \\
q(x, y, t)=p(x, h(x, t) y, t)
\end{array}\right\}
$$

The incompressibility condition takes then the form

$$
\operatorname{div}_{h}(\boldsymbol{u})=\frac{\partial u_{1}}{\partial x}-\frac{y}{h(x, t)} \frac{\partial h}{\partial x}(x, t) \frac{\partial u_{1}}{\partial y}+\frac{1}{h(x, t)} \frac{\partial u_{2}}{\partial y}+\frac{1}{h(x, t) y} u_{2}=0
$$

In the final stage of the proof, we are to send $\varepsilon \rightarrow 0$ in an integral identity that includes also terms

$$
\int_{0}^{T} \int_{D}\left\{\mu\left[\nabla_{h^{\varepsilon}} \boldsymbol{u}^{\varepsilon}+\left(\nabla_{h^{\varepsilon}} \boldsymbol{u}^{\varepsilon}\right)^{T}\right]: \nabla_{h^{\varepsilon}} \boldsymbol{\psi}^{\varepsilon}-q^{\varepsilon} \operatorname{div}_{h^{\varepsilon}} \boldsymbol{\psi}^{\varepsilon}\right\} \ell^{\varepsilon} d(x, y) d t,
$$

where

$$
\nabla_{h^{\varepsilon}} u_{i}^{\varepsilon}=\left(\frac{\partial u_{i}^{\varepsilon}}{\partial x}-\frac{y}{h^{\varepsilon}(x, t)} \frac{\partial h^{\varepsilon}}{\partial x}(x, t) \frac{\partial u_{i}^{\varepsilon}}{\partial y}, \frac{1}{h^{\varepsilon}(x, t)} \frac{\partial u_{i}^{\varepsilon}}{\partial y}\right)
$$


$\left(\boldsymbol{u}^{\varepsilon}, q^{\varepsilon}, \eta^{\varepsilon}\right)$ solve an appropriate collection of approximating problems and $\boldsymbol{\psi}^{\varepsilon}$ are test functions. The only way to overcome the lack of regularity for $\left\{q^{\varepsilon}\right\}$ is to consider divergencefree test functions, i.e.

$$
\operatorname{div}_{h^{\varepsilon}}\left(\psi^{\varepsilon}\right)=0
$$

This motivates us to find an explicit expression for a class of functions satisfying (1.18). For $\phi \in C^{3}(\bar{D} \times[0, T])$, let us define

$$
\left.\begin{array}{rl}
\psi_{1}^{\varepsilon}(x, y, t)= & -\frac{1}{\left(h^{\varepsilon}(x, t)\right)^{2}} \int_{0}^{x} \frac{\partial \phi}{\partial y}(\sigma, y, t) h^{\varepsilon}(\sigma, t) d \sigma, \\
\psi_{2}^{\varepsilon}(x, y, t)= & \phi(x, y, t)-\frac{1}{y} \int_{0}^{y} \phi(x, z, t) d z \\
& -\frac{y}{\left(h^{\varepsilon}(x, t)\right)^{2}} \frac{\partial h^{\varepsilon}}{\partial x}(x, t) \int_{0}^{x} \frac{\partial \phi}{\partial y}(\sigma, y, t) h^{\varepsilon}(\sigma, t) d \sigma
\end{array}\right\}
$$

and the desired equality (1.18) is valid for $\boldsymbol{\psi}^{\varepsilon}=\left(\psi_{1}^{\varepsilon}, \psi_{2}^{\varepsilon}\right)$. But now another difficulty arises, for, by inserting (1.19) into (1.17), the term

$$
\mu \int_{0}^{T} \int_{D}\left[\frac{\partial u_{2}^{\varepsilon}}{\partial x}-\frac{y}{h^{\varepsilon}} \frac{\partial h^{\varepsilon}}{\partial x} \frac{\partial u_{2}^{\varepsilon}}{\partial y}+\frac{1}{h^{\varepsilon}} \frac{\partial u_{1}^{\varepsilon}}{\partial y}\right] \frac{y}{\left(h^{\varepsilon}\right)^{2}} \frac{\partial^{2} h^{\varepsilon}}{\partial x^{2}} \int_{0}^{x} \frac{\partial \phi}{\partial y} h^{\varepsilon} d \sigma \ell^{\varepsilon} d(x, y) d t
$$

appears. The problem is that we cannot deduce more than weak convergence of the velocity gradient in $L^{2}$. What saves us are just the strong convergences of

$$
\frac{\partial^{2} h^{\varepsilon}}{\partial x^{2}} \rightarrow \frac{\partial^{2} h}{\partial x^{2}} \quad \text { in } L^{2}((0, L) \times(0, T)) \quad \text { and } \quad h^{\varepsilon} \rightarrow h \quad \text { in } W^{1, \infty}((0, L) \times(0, T)) .
$$

This example of $\boldsymbol{\psi}^{\varepsilon}$ implies that we in general need more regularity for $h^{\varepsilon}$, and therefore we will assume (1.15). Section 7 provides a detailed analysis of a limit process as $\varepsilon \rightarrow 0$ in the linearised perturbed NS equations.

Our study was originally initiated by the papers of Quarteroni [2-4] and it is in some sense a continuation of the paper [5]. Due to the fact that our main contribution is in introducing the cylindrical coordinate system to the fluid-structure interaction-like problem, we do not provide a representative list of references for 2-D problems. For a full summary of the research on solvability of the fluid-structure interaction problem, we refer the reader to the introduction in [1] and references therein. See also [6-8] and [9]. The methods that we apply borrow some material from [5, 10-12] and [13]; nevertheless, their application to our problem seems to be not straightforward.

The paper is organised as follows. In Section 2 we regularise once more our problem by considering it on a domain without the axis of symmetry (see, e.g. [14]) and then we transform it on the fixed domain. The main result of this section is the identity (2.21). Sections 3 and 5 provide necessary a priori estimates including the delicate identity (3.12). Sections 4 and 6 present main results of the paper. In Section 4, using the Schauder fixed point theorem, we prove the existence of the solution for the unknown interface, and in Section 6 we go to a limit with our regularised parameters. Finally, in Section 7 we let $\varepsilon \rightarrow 0$ in (1.2) but only for the linearised version of our problem, see (7.2). 
Throughout the paper we will always assume that there are positive constants $\alpha, K$, $0<\alpha \ll 1$, such that

$$
0<2 \alpha \leq h(x, t) \leq \alpha^{-1}, \quad\left|\frac{\partial h}{\partial x}(x, t)\right|,\left|\frac{\partial h}{\partial t}(x, t)\right| \leq K
$$

for all $(x, t) \in\left[-L^{-}, L^{+}\right] \times[0, T]$. Note that at the end we have to prove the validity of (1.20).

\section{Regularisation}

Our approach is to consider an approximate problem first. Given numbers

$$
0<\varepsilon \ll 1, \quad 0<\delta \ll \alpha, \quad \kappa \gg 1
$$

and given a function $h \in C^{1}\left(\left[-L^{-}, L^{+}\right] \times[0, T]\right)$ satisfying $(1.20)$, consider the system

$$
\left.\begin{array}{c}
\rho \frac{\partial v_{x}}{\partial t}+\rho v_{x} \frac{\partial v_{x}}{\partial x}+\rho v_{r} \frac{\partial v_{x}}{\partial r}+\frac{\rho}{2} v_{x}\left(\frac{\partial v_{x}}{\partial x}+\frac{\partial v_{r}}{\partial r}+\frac{1}{r} v_{r}\right) \\
=\mu\left\{2 \frac{\partial^{2} v_{x}}{\partial x^{2}}+\frac{1}{r} \frac{\partial}{\partial r}\left(r\left(\frac{\partial v_{x}}{\partial r}+\frac{\partial v_{r}}{\partial x}\right)\right)\right\}-\frac{\partial p}{\partial x}, \\
\rho \frac{\partial v_{r}}{\partial t}+\rho v_{x} \frac{\partial v_{r}}{\partial x}+\rho v_{r} \frac{\partial v_{r}}{\partial r}+\frac{\rho}{2} v_{r}\left(\frac{\partial v_{x}}{\partial x}+\frac{\partial v_{r}}{\partial r}+\frac{1}{r} v_{r}\right) \\
=\mu\left\{\frac{2}{r} \frac{\partial}{\partial r}\left(r \frac{\partial v_{r}}{\partial r}\right)+\frac{\partial}{\partial x}\left(\frac{\partial v_{r}}{\partial x}+\frac{\partial v_{x}}{\partial r}\right)-\frac{2}{r^{2}} v_{r}\right\}-\frac{\partial p}{\partial r}, \\
\varepsilon\left(\frac{\partial p}{\partial t}-\frac{\partial^{2} p}{\partial x^{2}}-\frac{1}{r} \frac{\partial}{\partial r}\left(r \frac{\partial p}{\partial r}\right)\right)+\frac{\partial v_{x}}{\partial x}+\frac{\partial v_{r}}{\partial r}+\frac{1}{r} v_{r}=0
\end{array}\right\}
$$

in

$$
\begin{aligned}
& \Omega^{\delta}(h)=\left\{(x, r, t):-L^{-}<x<L^{+}, \delta<r<h(x, t), 0<t<T\right\}, \\
& {\left[\frac{\partial^{2} \eta}{\partial t^{2}}+e \frac{\partial^{5} \eta}{\partial t^{3} \partial x^{2}}+d \frac{\partial^{5} \eta}{\partial t \partial x^{4}}+f \frac{\partial^{4} \eta}{\partial x^{4}}-c \frac{\partial^{3} \eta}{\partial t \partial x^{2}}-a \frac{\partial^{2} \eta}{\partial x^{2}}+b \eta\right](x, t)} \\
& =-\frac{\kappa}{E}\left(\frac{\partial \eta}{\partial t}(x, t)-v_{r}(x, h(x, t), t)\right), \quad 0<x<L, 0<t<T,
\end{aligned}
$$

equipped with the following boundary and initial conditions:

$$
v_{x}(x, h(x, t), t)=0
$$

for any $-L^{-}<x<L^{+}, 0<t<T$,

$$
v_{r}(x, h(x, t), t)=0
$$

for any $-L^{-} \leq x \leq 0$ and $L \leq x \leq L^{+}, 0<t<T$,

$$
\begin{aligned}
& h(x, t)\left[2 \mu \frac{\partial v_{r}}{\partial r}+\mu\left(\frac{\partial v_{r}}{\partial x}+\frac{\partial v_{x}}{\partial r}\right)\left(-\frac{\partial h}{\partial x}\right)-p+p_{w}-\frac{\rho}{2} v_{r}\left(v_{r}-\frac{\partial h}{\partial t}\right)\right](x, h(x, t), t) \\
& \quad=\kappa\left(\frac{\partial \eta}{\partial t}(x, t)-v_{r}(x, h(x, t), t)\right)
\end{aligned}
$$


for any $0<x<L, 0<t<T$,

$$
\varepsilon\left[\frac{\partial p}{\partial x}\left(-\frac{\partial h}{\partial x}\right)+\frac{\partial p}{\partial r}\right](x, h(x, t), t)=-\frac{\varepsilon}{2} \frac{\partial h}{\partial t}(x, t) p(x, h(x, t), t)
$$

for any $-L^{-}<x<L^{+}, 0<t<T$,

$$
\begin{aligned}
& \left(2 \mu \frac{\partial v_{x}}{\partial x}-p-p_{\text {out }}-\frac{\rho}{2}\left|v_{x}\right|^{2}\right)\left(L^{+}, r, t\right)=0, \\
& v_{r}\left(L^{+}, r, t\right)=0, \quad \varepsilon \frac{\partial p}{\partial x}\left(L^{+}, r, t\right)=0
\end{aligned}
$$

for any $\delta<r<R^{+}, 0<t<T$,

$$
\begin{aligned}
& \left(2 \mu \frac{\partial v_{x}}{\partial x}-p+p_{\text {in }}-\frac{\rho}{2}\left|v_{x}\right|^{2}\right)\left(-L^{-}, r, t\right)=0, \\
& v_{r}\left(-L^{-}, r, t\right)=0, \quad \varepsilon \frac{\partial p}{\partial x}\left(-L^{-}, r, t\right)=0
\end{aligned}
$$

for any $\delta<r<R^{-}, 0<t<T$,

$$
\frac{\partial v_{x}}{\partial r}(x, \delta, t)=0, \quad v_{r}(x, \delta, t)=0, \quad \varepsilon \frac{\partial p}{\partial r}(x, \delta, t)=0
$$

for any $-L^{-}<x<L^{+}, 0<t<T$,

$$
\left(v_{x}(x, r, 0), v_{r}(x, r, 0)\right)=(0,0), \quad \varepsilon p(x, r, 0)=0
$$

for any $\delta<r<h(x, 0),-L^{-}<x<L^{+}$,

$$
\eta(x, 0)=0, \quad \frac{\partial \eta}{\partial t}(x, 0)=0
$$

for any $0<x<L$ and, finally,

$$
\eta(0, t)=\frac{\partial \eta}{\partial x}(0, t)=\eta(L, t)=\frac{\partial \eta}{\partial x}(L, t)=0
$$

for any $0<t<T$. Note that in this section $h$ is given and $\left(v_{x}, v_{r}\right), p$ and $\eta$ are to be found ( $h$ and $\eta$ are therefore not related by (1.4)).

Assume for a moment that $\left(v_{x}, v_{r}, p, \eta\right)$ is in fact a smooth solution of problem (2.2)-(2.6) above. Then

$$
\left.\begin{array}{l}
\boldsymbol{u}(x, y, t) \stackrel{\text { def }}{=}\left(v_{x}(x,(h(x, t)-\delta) y+\delta, t), v_{r}(x,(h(x, t)-\delta) y+\delta, t)\right), \\
q(x, y, t) \stackrel{\text { def }}{=} p(x,(h(x, t)-\delta) y+\delta, t)
\end{array}\right\}
$$

for $0<x<L, 0<y<1,0<t<T$ and

$$
z(x, t) \stackrel{\text { def }}{=} \frac{\partial \eta}{\partial t}(x, t), \quad Z(x, t)=\int_{0}^{t} z(x, s) d s
$$


for $0<x<L, 0<t<T$ solve the following problem:

$$
\begin{aligned}
& \rho \frac{\partial(\ell \boldsymbol{u})}{\partial t}-\rho \frac{\partial h}{\partial t} \frac{\partial}{\partial y}\left(\frac{y \ell}{h-\delta} \boldsymbol{u}\right)+\rho \ell\left(\boldsymbol{u} \cdot \nabla_{h}\right) \boldsymbol{u}+\frac{\rho \ell}{2} \boldsymbol{u} \operatorname{div}_{h} \boldsymbol{u} \\
& \quad-\mu \ell \operatorname{div}_{h}\left(\nabla_{h} \boldsymbol{u}+\left(\nabla_{h} \boldsymbol{u}\right)^{T}\right)+\mu \frac{2(h-\delta)^{2}}{\ell}\left(\begin{array}{c}
0 \\
u_{2}
\end{array}\right) \\
& =-\ell \nabla_{h} q
\end{aligned}
$$

in $D \times(0, T)$, where $D=\left(-L^{-}, L^{+}\right) \times(0,1)$,

$$
\begin{aligned}
& \nabla_{h} u_{i}=\left(\frac{\partial u_{i}}{\partial x}+m \frac{\partial u_{i}}{\partial y}, \frac{1}{h-\delta} \frac{\partial u_{i}}{\partial y}\right), \\
& m(x, y, t)=-\frac{y}{h(x, t)-\delta} \frac{\partial h}{\partial x}(x, t), \\
& \operatorname{div}_{h}(\boldsymbol{u})=\frac{\partial u_{1}}{\partial x}+m \frac{\partial u_{1}}{\partial y}+\frac{1}{h-\delta} \frac{\partial u_{2}}{\partial y}+\frac{h-\delta}{\ell} u_{2}, \\
& \ell(x, y, t)=[(h(x, t)-\delta) y+\delta](h(x, t)-\delta), \\
& \varepsilon\left(\frac{\partial(h q)}{\partial t}-\frac{\partial h}{\partial t} \frac{\partial(y q)}{\partial y}-h \Delta_{h} q\right)+h \operatorname{div}_{h} \boldsymbol{u}=0, \quad \Delta_{h} q=\operatorname{div}_{h}\left(\nabla_{h} q\right),
\end{aligned}
$$

in $D \times(0, T)$,

$$
\begin{gathered}
\frac{\partial z}{\partial t}(x, t)+e \frac{\partial^{4} z}{\partial x^{2} \partial t^{2}}(x, t)+d \frac{\partial^{4} z}{\partial x^{4}}(x, t)+f \frac{\partial^{4} Z}{\partial x^{4}}(x, t) \\
-c \frac{\partial^{2} z}{\partial x^{2}}(x, t)-a \frac{\partial^{2} Z}{\partial x^{2}}(x, t)+b Z(x, t) \\
=-\frac{\kappa}{E}\left(z(x, t)-u_{2}(x, 1, t)\right)
\end{gathered}
$$

for any $0<x<L, 0<t<T$ with the boundary and initial conditions listed below:

$$
\begin{aligned}
& u_{1}(x, 1, t)=0 \quad \text { for any }-L^{-}<x<L^{+}, 0<t<T, \\
& u_{2}(x, 1, t)=0 \quad \text { for any }-L^{-}<x \leq 0 \text { and } L \leq x<L^{+}, 0<t<T, \\
& h\left[\frac{2 \mu}{h-\delta} \frac{\partial u_{2}}{\partial y}-\mu \frac{\partial h}{\partial x}\left(\frac{\partial u_{2}}{\partial x}+m \frac{\partial u_{2}}{\partial y}+\frac{1}{h-\delta} \frac{\partial u_{1}}{\partial y}\right)-q\right. \\
& \left.\quad+q_{w}-\frac{\rho u_{2}}{2}\left(u_{2}-\frac{\partial h}{\partial t}\right)\right](x, 1, t)=\kappa\left(z(x, t)-u_{2}(x, 1, t)\right), \\
& {\left[\frac{1}{h-\delta} \frac{\partial q}{\partial y}-\frac{\partial h}{\partial x}\left(\frac{\partial q}{\partial x}+m \frac{\partial q}{\partial y}\right)+\frac{1}{2} \frac{\partial h}{\partial t} q\right](x, 1, t)=0}
\end{aligned}
$$

for any $0<x<L, 0<t<T, q_{w}(x, t)=p_{w}(x, t)$,

$$
\begin{aligned}
& {\left[2 \mu\left(\frac{\partial u_{1}}{\partial x}+m \frac{\partial u_{1}}{\partial y}\right)-q+q_{\text {out }}-\frac{1}{2}|\boldsymbol{u}|^{2}\right]\left(L^{+}, y, t\right)=0,} \\
& u_{2}\left(L^{+}, y, t\right)=0, \quad\left(\frac{\partial q}{\partial x}+m \frac{\partial q}{\partial y}\right)\left(L^{+}, y, t\right)=0
\end{aligned}
$$


for any $0<y<1,0<t<T, q_{\text {out }}(y, t)=p_{\text {out }}\left(R^{+} y, t\right)$,

$$
\begin{aligned}
& {\left[2 \mu\left(\frac{\partial u_{1}}{\partial x}+m \frac{\partial u_{1}}{\partial y}\right)-q+q_{\text {in }}-\frac{1}{2}|\boldsymbol{u}|^{2}\right]\left(-L^{-}, y, t\right)=0,} \\
& u_{2}\left(-L^{-}, y, t\right)=0, \quad\left(\frac{\partial q}{\partial x}+m \frac{\partial q}{\partial y}\right)\left(-L^{-}, y, t\right)=0
\end{aligned}
$$

for any $0<y<1,0<t<T, q_{\text {in }}(y, t)=p_{\text {in }}\left(R^{-} y, t\right)$,

$$
\mu \frac{\partial u_{1}}{\partial y}(x, 0, t)=0, \quad u_{2}(x, 0, t)=0, \quad \varepsilon \frac{\partial q}{\partial y}(x, 0, t)=0
$$

for any $-L^{-}<x<L^{+}, 0<t<T$,

$$
\boldsymbol{u}(x, y, 0)=\mathbf{0}, \quad q(x, y, 0)=0
$$

for any $-L^{-}<x<L^{+}, 0<y<1$ and, finally,

$$
z(x, 0)=z(0, t)=\frac{\partial z}{\partial x}(0, t)=z(L, t)=\frac{\partial z}{\partial x}(L, t)=0
$$

for any $0<x<L$ and for any $0<t<T$.

We continue this section by making precise the meaning of the solution of problem (2.9)-(2.17). To this end, we first define

$$
V \equiv \boldsymbol{V} \times H^{1}(D) \times H_{0}^{2}(0, L)
$$

where

$$
\boldsymbol{V} \equiv\left\{\boldsymbol{w} \in H^{1}(D)^{2}: w_{1}=0 \text { on } S_{w}, w_{2}=0 \text { on } S_{\text {in }} \cup S_{\text {out }} \cup S_{c} \cup \Gamma\right\}
$$

and

$$
\begin{aligned}
& S_{w}=\left\{(x, 1):-L^{-}<x<L^{+}\right\}, \quad S_{\text {in }}=\left\{\left(-L^{-}, y\right): 0<y<1\right\}, \\
& S_{\text {out }}=\left\{\left(L^{+}, y_{2}\right): 0<y<1\right\}, \quad S_{c}=\left\{(x, 0):-L^{-}<x<L^{+}\right\}, \\
& \Gamma=\left\{(x, 1): x \in\left(-L^{-}, 0\right) \cup\left(L, L^{+}\right)\right\} \subset S_{w} .
\end{aligned}
$$

Throughout this and the next sections, we assume

$$
q_{\mathrm{in}}, q_{\mathrm{out}} \in L^{2}\left(0, T ; L^{2}(0,1)\right), \quad q_{w} \in L^{2}\left(0, T ; L^{2}(0, L)\right) .
$$

The function spaces we use are rather familiar, and we adopt the notation of [15].

Definition 2.1 We call $(\boldsymbol{u}, q, z) \in L^{2}(0, T ; V)$ a weak solution of the initial boundary value problem (2.9)-(2.17) if the following two properties are fulfilled:

(1) $\boldsymbol{u} \in L^{\infty}\left(0, T ; L^{2}(D)^{2}\right), q \in L^{\infty}\left(0, T ; L^{2}(D)\right), \frac{\partial(\ell q)}{\partial t} \in L^{2}\left(0, T ; H^{-1}(D)\right), \frac{\partial(\ell \boldsymbol{u})}{\partial t} \in\left(L^{2}(0\right.$, $\left.T ; \boldsymbol{V}) \cap L^{4}\left(0, T ; L^{4}(D)^{2}\right)\right)^{*}=L^{2}\left(0, T ; V^{*}\right)+L^{4 / 3}\left(0, T ; L^{4 / 3}(D)^{2}\right)$ and $z \in L^{\infty}\left(0, T ; H_{0}^{2}(0, L) \cap \cap\right.$ $H_{0-}^{1}\left(0, T ; H_{0}^{1}(0, L)\right)$. 
(2) $(\boldsymbol{u}, q, z)$ satisfies the system of differential equations, that is,

$$
\begin{aligned}
& \int_{0}^{T}\left\{\rho\left\langle\frac{\partial(\boldsymbol{u} \ell)}{\partial t}, \boldsymbol{\psi}\right\rangle+\varepsilon\left\langle\frac{\partial(q \ell)}{\partial t}, \omega\right\rangle\right\} d t \\
& -\frac{\rho}{2} \int_{0}^{T} \int_{D}\left\{(\boldsymbol{u} \cdot \boldsymbol{\psi}) \frac{\partial \ell}{\partial t}+\left[\frac{\partial \boldsymbol{u}}{\partial y} \cdot \boldsymbol{\psi}-\boldsymbol{u} \cdot \frac{\partial \boldsymbol{\psi}}{\partial y}\right][(h-\delta) y+\delta] \frac{\partial h}{\partial t} y\right\} d(x, y) d t \\
& -\frac{\varepsilon}{2} \int_{0}^{T} \int_{D}\left\{q \omega \frac{\partial \ell}{\partial t}+\left[\frac{\partial q}{\partial y} \omega-q \frac{\partial \omega}{\partial y}\right][(h-\delta) y+\delta] \frac{\partial h}{\partial t} y\right\} d(x, y) d t \\
& +\int_{0}^{T} \int_{D}\left\{\frac{\rho}{2}\left[\left(\left(\boldsymbol{u} \cdot \nabla_{h}\right) \boldsymbol{u}\right) \cdot \boldsymbol{\psi}-\left(\left(\boldsymbol{u} \cdot \nabla_{h}\right) \boldsymbol{\psi}\right) \cdot \boldsymbol{u}\right] \ell\right. \\
& +\mu\left(\left[\nabla_{h} \boldsymbol{u}+\left(\nabla_{h} \boldsymbol{u}\right)^{T}\right]: \nabla_{h} \boldsymbol{\psi}+\frac{2(h-\delta)^{2}}{\ell^{2}} u_{2} \psi_{2}\right) \ell \\
& \left.+\varepsilon\left(\nabla_{h} q \cdot \nabla_{h} \omega\right) \ell+\left(\omega \operatorname{div}_{h} \boldsymbol{u}-q \operatorname{div}_{h} \boldsymbol{\psi}\right) \ell\right\} d(x, y) d t \\
& +E \int_{0}^{T} \int_{0}^{L}\left\{\frac{\partial z}{\partial t} \xi+d \frac{\partial^{2} z}{\partial x^{2}} \frac{\partial^{2} \xi}{\partial x^{2}}+e \frac{\partial^{2} z}{\partial x \partial t} \frac{\partial^{2} \xi}{\partial x \partial t}+f \frac{\partial^{2} Z}{\partial x^{2}} \frac{\partial^{2} \xi}{\partial x^{2}}\right. \\
& \left.+c \frac{\partial z}{\partial x} \frac{\partial \xi}{\partial x}+a \frac{\partial Z}{\partial x} \frac{\partial \xi}{\partial x}+b Z \xi\right\} d x d t \\
& +\int_{0}^{T} \int_{0}^{L}\left\{q_{w} \psi_{2} h(h-\delta)+\kappa\left(u_{2}-z\right)\left(\psi_{2}-\xi\right)\right\} d x d t \\
& =\int_{0}^{T} \int_{0}^{1}\left\{q_{\text {in }}(y, t)\left(\psi_{1} \ell\right)\left(-L^{-}, y, t\right)+q_{\text {out }}(y, t)\left(\psi_{1} \ell\right)\left(L^{+}, y, t\right)\right\} d y d t
\end{aligned}
$$

for every $(\boldsymbol{\psi}, \omega, \xi) \in L^{2}(0, T ; V), \boldsymbol{\psi} \in L^{4}\left(0, T ; L^{4}(D)^{2}\right), \xi \in H^{1}\left(0, T ; H^{1}(0, L)\right) \cap L^{2}(0, T$; $\left.H_{0}^{2}(0, L)\right)$.

Remark 2.1 Note that

$$
\int_{0}^{T}\left\langle\frac{\partial(\ell \mathbf{u})}{\partial t}, \zeta\right\rangle d t+\int_{0}^{T} \int_{D} \ell \mathbf{u} \cdot \frac{\partial \zeta}{\partial t} d t=0
$$

for every test function $\zeta \in L^{2}(0, T ; \boldsymbol{V}) \cap L^{4}\left(0, T ; L^{4}(D)^{2}\right) \cap H^{1,1}\left(0, T ; L^{2}(D)^{2}\right)$ with $\zeta(T)=0$.

At the end of this section, we discuss the existence and uniqueness of weak solutions $(\boldsymbol{u}, q, z)$ to problem (2.9)-(2.17) for given $h, q_{w}, q_{\text {in }}$ and $q_{\text {out }}$ satisfying (1.20) and (2.20).

Theorem 2.1 Assume (2.1), (2.20), and let $\bar{\eta} \in C^{1}([0, L] \times[0, T])$ satisfying (1.7) and (1.8) be given. Hereafter $h=h(\bar{\eta})$ denotes $h$ defined by (1.4) with the given $\bar{\eta}$, and we assume that (1.20) holds. Then there exists a unique solution

$$
\mathcal{L}(\bar{\eta})=(\boldsymbol{u}, q, z)=\left(\boldsymbol{u}_{\varepsilon, \kappa, \delta}, q_{\varepsilon, \kappa, \delta}, z_{\varepsilon, \kappa, \delta}\right)
$$

of problem (2.9)-(2.17) in the sense of Definition 2.1 such that

$$
\int_{D}|\boldsymbol{u}|^{2}(t) \ell(t) d(x, y)+\int_{0}^{t} \int_{D}|\boldsymbol{u}|^{2} \frac{\partial \ell}{\partial t} d(x, y) d s=2 \int_{0}^{t}\left\langle\frac{\partial(\boldsymbol{u} \ell)}{\partial t}, \boldsymbol{u}\right\rangle d s,
$$


where the function $\ell$ in (2.23) is defined by (2.10) for $h=h(\bar{\eta})$, i.e.

$$
\ell(x, y, t)=[(h(x, t)-\delta) y+\delta](h(x, t)-\delta) .
$$

Proof of Theorem 2.1 By analogy with the approach taken in [5, the proof of Theorem 6.3], we can construct our weak solution by the implicit time discretisation method. The paper [5] presents all the technicalities of the proof, therefore we omit the proof here.

We conclude this section by some interpolation inequalities which are needed especially for estimating the nonlinear item. For this purpose, we complete the notations of function spaces (2.18) and (2.19) by appropriate weighted spaces. Let

$$
\begin{aligned}
& L_{y}^{p}(D) \equiv\left\{\phi: D \rightarrow \mathbb{R}: \int_{D}|\phi(x, y)|^{p} y d(x, y)<\infty\right\}, \\
& H_{y}^{1, p}(D) \equiv\left\{\omega \in L_{y}^{p}(D): \nabla \omega \in L_{y}^{p}(D)\right\}, \quad H_{y}^{1}(D) \equiv H_{y}^{1,2}(D)
\end{aligned}
$$

and

$$
\begin{aligned}
\boldsymbol{V}_{y} \equiv & \left\{\boldsymbol{w} \in L_{y}^{2}(D)^{2}:|\nabla \boldsymbol{w}| \in L_{y}^{2}(D), y^{-1 / 2} w_{2} \in L_{y}^{2}(D),\right. \\
& \left.w_{1}=0 \text { on } S_{w}, w_{2}=0 \text { on } S_{\text {in }} \cup S_{\text {out }} \cup S_{c}\right\}, \\
V_{y} \equiv & \boldsymbol{V}_{y} \times H_{y}^{1}(D) \times H_{0}^{2}(0, L) .
\end{aligned}
$$

Proposition 2.1 (i) Let $\varphi$ be any function in $H^{1}(D)$ such that $\varphi=0$ on $S_{w}$ or $\varphi=0$ on $S_{c}$. Then, for any $p \geq 2$ and for any number $\theta$ with

$$
\frac{p-2}{p} \leq \theta \leq 1
$$

there exists a constant $C=C(p, \theta)$ such that

$$
\|\varphi\|_{L^{p}(D)} \leq C\|\nabla \varphi\|_{L^{2}(D)}^{\theta}\|\varphi\|_{L^{2}(D)}^{1-\theta}
$$

Moreover, if $\varphi \in L^{2}\left(0, T ; H^{1}(D)\right) \cap L^{\infty}\left(0, T ; L^{2}(D)\right)$ such that $\varphi=0$ on $S_{w}$ or $\varphi=0$ on $S_{c}$ for almost all $t \in[0, T]$, then the following holds for any $p \geq 2$ :

$$
\|\varphi\|_{L^{\frac{2 p}{p-2}\left(0, T ; L^{p}(D)\right)}} \leq C\left(\|\varphi\|_{L^{\infty}\left(0, T ; L^{2}(D)\right)}\right)^{2 / p}\left(\|\varphi\|_{L^{2}\left(0, T ; H^{1}(D)\right)}\right)^{(p-2) / p} .
$$

(ii) Let now $p \geq 2$ and $\theta \leq 1$ fulfil the condition

$$
\frac{3}{2} \frac{p-2}{p} \leq \theta \leq 1
$$

Then there exists a constant $C=C(p, \theta)$ such that

$$
\|\boldsymbol{w}\|_{L_{y}^{p}(D)^{2}} \leq C\|\nabla \boldsymbol{w}\|_{L_{y}^{2}(D)^{2}}^{\theta}\|\boldsymbol{w}\|_{L_{y}^{2}(D)^{2}}^{1-\theta} \quad \text { for any } \boldsymbol{w} \in \boldsymbol{V}_{y} .
$$


If, moreover, $\boldsymbol{w} \in L^{2}\left(0, T ; H_{y}^{1}(D)^{2}\right) \cap L^{\infty}\left(0, T ; L_{y}^{2}(D)^{2}\right)$ such that $w_{1}=0$ on $S_{c}$ and $w_{2}=0$ on $S_{w}$ for almost all $t \in[0, T]$, then the following holds for any $p \in[2,6]$ :

$$
\begin{aligned}
& \|\boldsymbol{w}\|_{L^{\frac{4 p}{3(p-2)}}\left(0, T ; L_{y}^{p}(D)^{2}\right)} \\
& \quad \leq C\left(\|\boldsymbol{w}\|_{L^{\infty}\left(0, T ; L_{y}^{2}(D)^{2}\right)}\right)^{(6-p) / 2 p}\left(\|\boldsymbol{w}\|_{L^{2}\left(0, T ; H_{y}^{1}(D)^{2}\right)}\right)^{(3 p-6) / 2 p} .
\end{aligned}
$$

Proof (i) The form of Nirenberg-Gagliardo inequality (2.26) can be found, e.g. in [16, Theorem 2.2]. Then (2.27) follows from (2.26) for $\theta=(p-2) / p$ by integration over $(0, T)$.

(ii) To prove (2.28), we define $\widetilde{\psi}\left(x, y_{2}, y_{3}\right):=\psi\left(x, \sqrt{y_{2}^{2}+y_{3}^{2}}\right)$ and transform the integrals on the domain $D$ into integrals on the cylinder $\widetilde{D}=\left\{\left(x, y_{2}, y_{3}\right) \in \mathbb{R}^{3}:-L^{-}<x<L^{+}, y_{2}^{2}+\right.$ $y_{3}^{2}<1$. Then the weighted interpolation inequality (2.28) is equivalent to an unweighted interpolation inequality in $\mathbb{R}^{3}$ for $\widetilde{\psi}$ given on $\widetilde{D}$, which yields the assertion ( $c f$. also [17, Theorem 19.9] for $\theta=1)$. Inequality (2.29) again follows by integration of (2.28) over $t \in$ $(0, T)$ with $\theta=3(p-2) / 2 p$.

\section{The first a priori estimate}

Our ultimate goal is to show that a subsequence of solutions $\left(\boldsymbol{u}_{\varepsilon, \kappa, \delta}, q_{\varepsilon, \kappa, \delta}, z_{\varepsilon, \kappa, \delta}\right)$ of the approximate problems (2.9)-(2.17) converges to a weak solution of (1.1)-(1.14). For this we will need some uniform estimates. Let us start with the following theorem.

Theorem 3.1 Let the hypotheses of Theorem 2.1 be satisfied, and let $\mathcal{L}(\bar{\eta})=(\boldsymbol{u}, q, z)$ be the corresponding solution of problem (2.9)-(2.17).

Then there exists a constant $C$ such that

$$
\begin{aligned}
& \int_{D}\left(\rho|\boldsymbol{u}(t)|^{2}+\varepsilon|q(t)|^{2}\right) \ell(t) d(x, y) \\
& +\frac{\alpha^{2}}{2\left(K^{2}+2\right)} \int_{0}^{t} \int_{D}\left(\mu|\nabla \mathbf{u}|^{2}+\varepsilon|\nabla q|^{2}\right) \ell d(x, y) d s \\
& \quad+2 \mu \int_{0}^{t} \int_{D} \frac{(h-\delta)^{2}}{\ell}\left|u_{2}\right|^{2} d(x, y) d s \\
& \quad+E \int_{0}^{L}\left(\left|Z_{t}\right|^{2}+f\left|Z_{x x}\right|^{2}+a\left|Z_{x}\right|^{2}+b|Z|^{2}\right)(x, t) d x \\
& \quad+2 E \int_{0}^{t} \int_{0}^{L}\left(d\left|Z_{t x x}\right|^{2}+e\left|Z_{t x t}\right|^{2}+c\left|Z_{t x}\right|^{2}\right) d x d s \\
& \quad+2 \kappa \int_{0}^{t} \int_{0}^{L}\left|u_{2}(x, 1, s)-z(x, s)\right|^{2} d x d s \\
& \leq \alpha^{-2} M_{q_{b}}^{2}(t)\left[1+e^{F t} F t\right]
\end{aligned}
$$

for a.e. time $0 \leq t \leq T$, where

$$
\begin{aligned}
& M_{q_{b}}^{2}(t)=\int_{0}^{t} \int_{0}^{L}\left|q_{w}\right|^{2} d x d s+\int_{0}^{t} \int_{0}^{1}\left(\left|q_{\text {in }}\right|^{2}+\left|q_{\text {out }}\right|^{2}\right) y d y d s, \\
& F=\frac{C\left(K^{2}+2\right)}{\alpha^{2} \mu \rho} \quad \text { and } \quad Z_{t}=\frac{\partial Z}{\partial t}=z, \quad Z_{x x}=\frac{\partial^{2} Z}{\partial x^{2}}, \quad \ldots
\end{aligned}
$$

The constant $C$ does not depend on $\alpha, K, \varepsilon, \kappa$ and $\delta$. 
Before proving Theorem 3.1, we pause to introduce some of its consequences.

Theorem 3.2 Let $\boldsymbol{u}=\left(u_{1}, u_{2}\right)=\boldsymbol{u}_{\varepsilon, \kappa, \delta}$ and $\ell=\ell_{\delta}$. According to the energy estimates (3.1), we see that the set

$$
\left\{\ell^{\sigma} \boldsymbol{u}\right\} \quad \text { is bounded in } L^{\infty}\left(0, T ; L^{2}(D)^{2}\right) \cap L^{2}(0, T ; \boldsymbol{V})
$$

for any $\sigma \geq 3 / 2$, the set

$$
\left\{\sqrt{\ell} u_{2}\right\} \quad \text { is bounded in } L^{\infty}\left(0, T ; L^{2}(D)\right) \cap L^{2}\left(0, T ; H^{1}(D)\right)
$$

and the set

$$
\left\{\sqrt{\ell} u_{1}\right\} \quad \text { is bounded in } L^{\infty}\left(0, T ; L^{2}(D)\right)
$$

for all $\varepsilon, \kappa, \delta$. Moreover, we obtain that

$$
\left\{\ell^{(\lambda / 2)} \mathbf{u}\right\} \text { and }\left\{y^{(\lambda / 2)} \mathbf{u}\right\} \text { are bounded in } L^{2}\left(0, T ; L^{2}(D)^{2}\right)
$$

for any fixed $\lambda>-1$ and all $\varepsilon, \kappa, \delta$ satisfying (2.1).

Proof of Theorem 3.2 It remains to prove (3.5) and it suffices to prove the assertion for the first component $u_{1}$ of $\boldsymbol{u}$ since for $u_{2}$ it follows from estimate (3.1). For a moment, let us fix $x$ and $t$ and set $u(y)=u_{1}(x, y, t), \ell(y)=\ell(x, y, t)$. As $u(1)=0$, we have

$$
|u(y)|^{2}=\left|\int_{y}^{1} \frac{u^{\prime}(s) \sqrt{s}}{\sqrt{s}} d s\right|^{2} \leq-\ln y \int_{0}^{1}\left|u^{\prime}(s)\right|^{2} s d s
$$

and

$$
\alpha^{2} y \leq \ell(y) \leq 1+\alpha^{-2}
$$

Now we shall distinguish two cases. In order to derive (3.5) in the case $-1<\lambda<0$, let us multiply (3.6) by $\alpha^{2 \lambda} y^{\lambda}$ and integrate it over $(0,1)$. Due to (3.7), this gives the relation

$$
\begin{aligned}
\int_{0}^{1}|u(y)|^{2} \ell^{\lambda}(y) d y & \leq \alpha^{2 \lambda} \int_{0}^{1}|u(y)|^{2} y^{\lambda} d y \leq \frac{\alpha^{2 \lambda}}{(\lambda+1)^{2}} \int_{0}^{1}\left|u^{\prime}(s)\right|^{2} s d s \\
& \leq \frac{\alpha^{2(\lambda-1)}}{(\lambda+1)^{2}} \int_{0}^{1}\left|u^{\prime}(s)\right|^{2} \ell(s) d s
\end{aligned}
$$

and (3.5) follows by integration over $\left(-L^{-}, L^{+}\right) \times(0, T)$. If $\lambda \geq 0$, then

$$
\begin{aligned}
\alpha^{2 \lambda} \int_{0}^{1}|u(y)|^{2} y^{\lambda} d y & \leq \int_{0}^{1}|u(y)|^{2} \ell^{\lambda}(y) d y \\
& \leq\left(1+\alpha^{-2}\right)^{\lambda}\left(-\int_{0}^{1} \ln y d y\right)\left(\int_{0}^{1}\left|u^{\prime}(s)\right|^{2} s d s\right)
\end{aligned}
$$

and (3.5) follows easily. 
Note that (3.8) is a special case of the Hardy inequality [17, Theorem 6.2]. Moreover, we remark that this argument also applies to $q$, which in analogue to (3.5) yields that

$$
\left\{\ell^{(\lambda / 2)} q_{\varepsilon, \kappa, \delta}\right\} \quad \text { and } \quad\left\{y^{(\lambda / 2)} q_{\varepsilon, \kappa, \delta}\right\} \quad \text { are bounded in } L^{2}\left(0, T ; L^{2}(D)\right)
$$

for any fixed $\lambda>-1$.

Proof of Theorem 3.1 Fix now a positive $t<T$ and substitute $(\boldsymbol{\psi}, \omega, \xi)=(\boldsymbol{u}, q, z)$ into the identity (2.21). This is legitimate since $\boldsymbol{u} \in L^{4}\left(0, T ; L^{4}(D)^{2}\right)$. Then, with the assistance of (2.23), we write the resulting expression as

$$
\begin{aligned}
\rho \int_{D}|\boldsymbol{u}(t)|^{2} \ell(t) d(x, y)+\varepsilon \int_{D}|q(t)|^{2} \ell(t) d(x, y) \\
\quad+2 \mu \int_{0}^{t} \int_{D}\left\{\left[\nabla_{h} \boldsymbol{u}+\left(\nabla_{h} \boldsymbol{u}\right)^{T}\right]: \nabla_{h} \boldsymbol{u}+2\left(\frac{h-\delta}{\ell} u_{2}\right)^{2}\right\} \ell d(x, y) d s \\
\quad+2 \varepsilon \int_{0}^{t} \int_{D}\left|\nabla_{h} q\right|^{2} \ell d(x, y) d s \\
+E \int_{0}^{L}\left\{|z|^{2}+f\left|Z_{x x}\right|^{2}+a\left|Z_{x}\right|^{2}+b|Z|^{2}\right\}(t) d x \\
\quad+2 E \int_{0}^{t} \int_{0}^{L}\left\{d\left|z_{x x}\right|^{2}+e\left|z_{x t}\right|^{2}+c\left|z_{x}\right|^{2}\right\} d x d s \\
\quad+2 \kappa \int_{0}^{t} \int_{0}^{L}\left|u_{2}(x, 1, s)-z(x, s)\right|^{2} d x d s \\
=2 \int_{0}^{t} \int_{0}^{L} q_{w}(x, s) u_{2}(x, 1, s) \ell(x, 1, s) d x d s \\
+2 \int_{0}^{t} \int_{0}^{1}\left[\left(q_{\text {in }} \ell u_{1}\right)\left(-L^{-}, y, s\right)+\left(q_{\text {out }} \ell u_{1}\right)\left(L^{+}, y, s\right)\right] d y d s .
\end{aligned}
$$

Our plan now is to check the ellipticity condition that follows from the following two propositions.

\section{Proposition 3.1 Suppose}

$$
\boldsymbol{u} \in \boldsymbol{V}=\left\{\boldsymbol{w} \in H^{1}(D)^{2}: w_{1}=0 \text { on } S_{w}, w_{2}=0 \text { on } S_{\text {in }} \cup S_{\text {out }} \cup S_{c} \cup \Gamma\right\}
$$

see (2.19). Then

$$
\begin{aligned}
2 \mu \int_{D}\left\{\left[\nabla_{h} \boldsymbol{u}+\left(\nabla_{h} \boldsymbol{u}\right)^{T}\right]: \nabla_{h} \boldsymbol{u}+2\left(\frac{h-\delta}{\ell} u_{2}\right)^{2}\right\} \ell d(x, y) d t \\
=\mu \int_{D}\left\{3\left(\frac{\partial u_{1}}{\partial x}+m \frac{\partial u_{1}}{\partial y}\right)^{2}+\left(\frac{1}{h-\delta} \frac{\partial u_{1}}{\partial y}\right)^{2}\right. \\
+\left(\frac{\partial u_{2}}{\partial x}+m \frac{\partial u_{2}}{\partial y}\right)^{2}+2\left(\frac{1}{h-\delta} \frac{\partial u_{2}}{\partial y}\right)^{2}+2\left(\frac{h-\delta}{\ell} u_{2}\right)^{2} \\
+\left(\operatorname{div}_{h} \boldsymbol{u}\right)^{2}+\left(\frac{h-\delta}{\ell} u_{2}-\frac{1}{h-\delta} \frac{\partial u_{2}}{\partial y}\right)^{2} \\
\left.+\left(\frac{\partial u_{2}}{\partial x}+m \frac{\partial u_{2}}{\partial y}+\frac{1}{h-\delta} \frac{\partial u_{1}}{\partial y}\right)^{2}\right\} \ell d(x, y) .
\end{aligned}
$$


Proof of Proposition 3.1 Let us write the left-hand side of (3.12) as a sum of two integrals

$$
\begin{aligned}
& \mu \int_{D}\left\{\nabla_{h} \boldsymbol{u}: \nabla_{h} \boldsymbol{u}+2\left(\frac{h-\delta}{\ell} u_{2}\right)^{2}+\left(\nabla_{h} \boldsymbol{u}\right)^{T}: \nabla_{h} \boldsymbol{u}\right\} \ell d(x, y) d t \\
& +\mu \int_{D}\left\{\left[\nabla_{h} \boldsymbol{u}+\left(\nabla_{h} \boldsymbol{u}\right)^{T}\right]: \nabla_{h} \boldsymbol{u}+2\left(\frac{h-\delta}{\ell} u_{2}\right)^{2}\right\} \ell d(x, y) d t
\end{aligned}
$$

and we deduce that it equals

$$
\begin{aligned}
& \mu \int_{D}\left\{\nabla_{h} \boldsymbol{u}: \nabla_{h} \boldsymbol{u}+2\left(\frac{h-\delta}{\ell} u_{2}\right)^{2}+\left(\frac{\partial u_{1}}{\partial x}+m \frac{\partial u_{1}}{\partial y}\right)^{2}\right. \\
& \left.\quad+\frac{2}{h-\delta} \frac{\partial u_{1}}{\partial y}\left(\frac{\partial u_{2}}{\partial x}+m \frac{\partial u_{2}}{\partial y}\right)+\left(\frac{1}{h-\delta} \frac{\partial u_{2}}{\partial y}\right)^{2}\right\} \ell d(x, y) \\
& \quad+\mu \int_{D}\left\{2\left(\frac{\partial u_{1}}{\partial x}+m \frac{\partial u_{1}}{\partial y}\right)^{2}+\frac{1}{h-\delta} \frac{\partial u_{1}}{\partial y}\left(\frac{\partial u_{2}}{\partial x}+m \frac{\partial u_{2}}{\partial y}+\frac{1}{h-\delta} \frac{\partial u_{1}}{\partial y}\right)\right. \\
& \quad+\left(\frac{\partial u_{2}}{\partial x}+m \frac{\partial u_{2}}{\partial y}\right)\left(\frac{\partial u_{2}}{\partial x}+m \frac{\partial u_{2}}{\partial y}+\frac{1}{h-\delta} \frac{\partial u_{1}}{\partial y}\right) \\
& \left.\quad+2\left(\frac{1}{h-\delta} \frac{\partial u_{2}}{\partial y}\right)^{2}+2\left(\frac{h-\delta}{\ell} u_{2}\right)^{2}\right\} \ell d(x, y) .
\end{aligned}
$$

Now, let us focus on the first term in the second line of (3.13).

\section{Lemma 3.3 Assume $\mathbf{u} \in \boldsymbol{V}$, then}

$$
\begin{aligned}
\int_{D} \frac{\ell}{h-\delta} \frac{\partial u_{1}}{\partial y} \frac{\partial u_{2}}{\partial x} d(x, y)= & \int_{D} \frac{\ell}{h-\delta} \frac{\partial u_{1}}{\partial x} \frac{\partial u_{2}}{\partial y} d(x, y) \\
& +\int_{D}\left(\frac{\partial u_{1}}{\partial x}+m \frac{\partial u_{1}}{\partial y}\right)(h-\delta) u_{2} d(x, y)
\end{aligned}
$$

Proof of Lemma 3.3 Assume for a moment that $u_{1} \in H^{2}(D)$. After integrating by parts, we obtain

$$
\begin{aligned}
\int_{D} \frac{\ell}{h-\delta} \frac{\partial u_{1}}{\partial y} \frac{\partial u_{2}}{\partial x} d(x, y) & =-\int_{D} y \frac{\partial h}{\partial x} \frac{\partial u_{1}}{\partial y} u_{2} d(x, y)-\int_{D} \frac{\ell}{h-\delta} \frac{\partial^{2} u_{1}}{\partial y \partial x} u_{2} d(x, y) \\
& =\int_{D}\left(-y \frac{\partial h}{\partial x} \frac{\partial u_{1}}{\partial y} u_{2}+\frac{\partial}{\partial y}\left(\frac{\ell}{h-\delta} u_{2}\right) \frac{\partial u_{1}}{\partial x}\right) d(x, y)
\end{aligned}
$$

and (3.14) follows easily. The validity of (3.14) for $\boldsymbol{u} \in \boldsymbol{V}$ is a consequence of the approximation argument.

We apply now Lemma 3.3 to the first term on the second line of (3.13) and the validity of (3.12) can be verified by direct computations. Let us note, however, that it is rather tedious.

The proof of the next proposition can be found in [5, Lemma 4.3]. 
Proposition 3.2 Let the hypotheses of Theorem 2.1 be satisfied, and let $\boldsymbol{u} \in \boldsymbol{V}$, then

$$
\begin{aligned}
& \sum_{i=1}^{2} \mu \int_{D}\left[\left(\frac{\partial u_{i}}{\partial x}+m \frac{\partial u_{i}}{\partial y}\right)^{2}+\left(\frac{1}{h-\delta} \frac{\partial u_{i}}{\partial y}\right)^{2}\right] \ell d(x, y) \\
& \quad \geq \frac{\alpha^{2} \mu}{K^{2}+2} \int_{D}|\nabla \boldsymbol{u}|^{2} \ell d(x, y)
\end{aligned}
$$

We would now like to finish the proof of Theorem 3.1. Returning to the relation (3.11), we estimate its second line with the assistance of (3.12), (3.15) and its right-hand side by Hölder's inequality to find

$$
\begin{aligned}
\xi(t) & +\frac{\alpha^{2} \mu}{K^{2}+2} \int_{0}^{t} \int_{D}|\nabla \mathbf{u}|^{2} \ell d(x, y) d s \\
\leq & \alpha^{-2} M_{q_{b}}^{2}(t)+\int_{0}^{t} \int_{0}^{L}\left(\left|u_{2}\right|^{2} \ell\right)(x, 1, s) d x d s \\
& +\int_{0}^{t} \int_{0}^{1}\left[\left(\left|u_{1}\right|^{2} \ell\right)\left(-L^{-}, y, s\right)+\left(\left|u_{1}\right|^{2} \ell\right)\left(L^{+}, y, s\right)\right] d y d s
\end{aligned}
$$

for $\xi(t)=\rho \int_{D}\left(|\boldsymbol{u}|^{2} \ell\right)(x, y, t) d(x, y)$. Next, observe that for $\boldsymbol{u} \in \boldsymbol{V}$, the last two terms on the right-hand side of (3.16) are bounded by

$$
\iota \int_{0}^{t} \int_{D}|\nabla \mathbf{u}|^{2} \ell d(x, y) d s+\frac{C}{\iota} \int_{0}^{t} \xi(s) d s
$$

for any $0<\iota \ll 1$ and therefore also for $\iota=\alpha^{2} \mu /\left(2\left(K^{2}+2\right)\right)$. Consequently,

$$
\xi(t) \leq \alpha^{-2} M_{q_{b}}^{2}(t)+F \int_{0}^{t} \xi(s) d s
$$

for a.e. $0 \leq t \leq T$. Thus Gronwall's inequality yields the estimate

$$
\int_{0}^{t} \xi(s) d s \leq e^{F t} \int_{0}^{t} \alpha^{-2} M_{q b}^{2}(s) d s
$$

that due to (3.16) and (3.17) implies (3.1).

\section{A free boundary value problem}

So far we have studied problem (2.9)-(2.17) for $h=h(\bar{\eta})$ with the given function $\bar{\eta}$, and Theorem 2.1 gives the solution $\mathcal{L}(\bar{\eta})=(\boldsymbol{u}, q, z)$. For this section, keeping still $\varepsilon, \kappa, \delta$ fixed, we turn our attention to the problem of finding a function $\eta$ such that

$$
\eta(x, t)=\int_{0}^{t} z(x, s) d s \quad \text { for } z \text { given by } \mathcal{L}(\eta)=(\boldsymbol{u}, q, z)
$$

Our plan is hereafter to formulate it as a fixed point problem.

Thus, let $\mathcal{L}(\bar{\eta})=(\boldsymbol{u}, q, z)$ be the corresponding solution of problem (2.9)-(2.17). Then the first a priori estimate (3.1) suggests that bounds (1.20) should be valid for $h=h(Z)$ if 
positive $T$ or the norms of outer pressure are chosen sufficiently small provided $h=h(\bar{\eta})$ satisfies (1.20). Indeed, let us denote

$$
\mathcal{M}(T)=H_{0-}^{1}\left(0, T ; H_{0}^{2}(0, L)\right) \cap H_{0-}^{2}\left(0, T ; H_{0}^{1}(0, L)\right),
$$

and for $\xi \in \mathcal{M}(T)$,

$$
\|\xi\|_{T}^{2}=2 E \int_{0}^{T} \int_{0}^{L}\left(d\left|\xi_{x t x}\right|^{2}+e\left|\xi_{t x t}\right|^{2}\right) d x d t
$$

In view of Theorem 3.1 and the embeddings

$$
\mathcal{M}(T) \subset C^{1,1 / 2}\left(\overline{\mathcal{S}}_{T}\right) \subset \subset C^{1}\left(\overline{\mathcal{S}}_{T}\right), \quad\|\xi\|_{C^{1,1 / 2}\left(\bar{S}_{T}\right)} \leq \mathcal{C}_{e}(T)\|\xi\|_{T}
$$

(note that $\mathcal{C}_{e}(T)$ is increasing in $T$ ), we have the following theorem.

Theorem 4.1 Suppose that the data satisfy (2.20), and assume $0<\alpha \ll 1$ such small that

$$
4 \alpha<R^{+}+R^{-}<\alpha^{-1}, \text { and let } K=R^{+}-2 \alpha+\left\|\chi^{\prime}\right\|_{\infty} .
$$

Fix any point $\bar{\eta} \in C^{1}\left(\overline{\mathcal{S}}_{T}\right)$ such that $h=h(\bar{\eta})$ satisfies (1.20) with $\alpha$ and $K$ given above. Let us recall that $\mathcal{L}(\bar{\eta})=(\boldsymbol{u}, q, z)$ is the solution of problem (2.9)-(2.17) with $h=h(\bar{\eta})$ in the sense of Theorem 2.1. If we recall the notation of (2.8), then

$$
\|Z\|_{C^{1,1 / 2}\left(\overline{\mathcal{S}}_{T}\right)} \leq \mathcal{C}_{e}(T)\|Z\|_{T} \leq R^{+}-2 \alpha
$$

provided $M_{q_{b}}(T)$ is sufficiently small. Moreover, $h=h(Z)$ satisfies (1.20) with the given $\alpha$ and $K$.

Note that smallness of $M_{q_{b}}(T)$ ( $c f$. Theorem 3.1) can be achieved by small outer pressure or a small time interval, but from now on, let $T$ be fixed.

Proof It remains to prove that $h=h(Z)$ satisfies (1.20). Because of $0<R^{+} \leq R^{-}$and $|Z| \leq$ $R^{+}-2 \alpha$, the following holds:

$$
2 \alpha \leq R^{+}-|Z(x, t)| \leq h(x, t) \leq R^{-}+|Z(x, t)| \leq R^{-}+R^{+} \leq \alpha^{-1} .
$$

The second estimate in (1.20) obviously follows from (4.4) by the definition of $h$ and the choice of $K$.

Denote now $X=C^{1}\left(\overline{\mathcal{S}}_{T}\right)$ and

$$
\mathcal{K}=\left\{\varphi \in \mathcal{M}(T):\|\varphi\|_{C^{1,1 / 2}\left(\overline{\mathcal{S}}_{T}\right)} \leq R^{+}-2 \alpha\right\} .
$$

Then $\mathcal{K} \subset X$ is compact and convex. Now we define a mapping

$$
\mathcal{A}: \mathcal{K} \rightarrow \mathcal{K}
$$


in the following way: We start with $\bar{\eta} \in \mathcal{K}$ and define $h=h(\bar{\eta})$ corresponding to formula (1.4), i.e. by

$$
h(\bar{\eta})(x, t)= \begin{cases}R^{-}, & -L^{-} \leq x \leq 0 \\ \chi(x)+\bar{\eta}(x, t), & 0 \leq x \leq L, \\ R^{+}, & L \leq x \leq L^{+} .\end{cases}
$$

Now, let $\mathcal{L}(\bar{\eta})=(\boldsymbol{u}, q, z)$ be the solution of problem (2.9)-(2.17) according to Definition 2.1 with $h=h(\bar{\eta})$, and let us define a mapping $\mathcal{P}(\boldsymbol{u}, q, z):=Z$.

Then our fixed point operator $\mathcal{A}$ is defined by $\mathcal{A}=\mathcal{P} \circ \mathcal{L}$, i.e.

$$
\mathcal{A}[\bar{\eta}]=Z
$$

Corollary 4.2 $\mathcal{A}$ is well defined on $\mathcal{K}$ and maps $\mathcal{K}$ into itself.

Proof Note that $h=h(\bar{\eta})$ fulfils (1.20) if $\bar{\eta} \in \mathcal{K}$ in view of Theorem 4.1. Then Theorem 2.1 implies that the mapping $\mathcal{L}: C^{1}\left(\bar{S}_{T}\right) \supset \mathcal{K} \rightarrow L^{2}(0, T ; V)$ is well defined and unique. Moreover, due to Theorem 3.1, $Z=\mathcal{P}(\boldsymbol{u}, q, z)$ belongs to $\mathcal{M}(T)$ with the finite norm $\|Z\|_{T}$ defined by (4.2). Finally, estimate (4.4) in Theorem 4.1 yields $Z=(\mathcal{P} \circ \mathcal{L})[\bar{\eta}] \subset \mathcal{K}$.

An application of the Schauder fixed point theorem requires the continuity of our mapping $\mathcal{A}$. This means that we need continuous dependence of solutions on the data.

Theorem 4.3 Assume (2.1). Let $\left(\boldsymbol{u}^{1}, q^{1}, z^{1}\right)$ and $\left(\boldsymbol{u}^{2}, q^{2}, z^{2}\right)$ be weak solutions of the initial boundary value problem (2.9)-(2.17) in the sense of Definition 2.1 with given functions $h^{1}=$ $h\left(\bar{\eta}^{1}\right), q_{\mathrm{in}}^{1}, q_{w}^{1}, q_{\mathrm{out}}^{1}$ and $h^{2}=h\left(\bar{\eta}^{2}\right), q_{\mathrm{in}}^{2}, q_{w}^{2}, q_{\mathrm{out}}^{2}$, respectively, and suppose that both $h^{1}, h^{2}$ satisfy (1.20). Let, moreover, $\tau, t \in[0, T], 0 \leq \tau<t$, with $\left(\boldsymbol{u}^{1}, q^{1}, z^{1}\right)(\cdot, s)=\left(\boldsymbol{u}^{2}, q^{2}, z^{2}\right)(\cdot, s)$ and $\bar{\eta}^{1}(\cdot, s)=\bar{\eta}^{2}(\cdot, s)$ for all $s \in[0, \tau]$. Then there exist positive constants $v_{u}$ and $v_{q}$ such that

$$
\begin{aligned}
\rho \int_{D} \mid & \ell^{1} \boldsymbol{u}^{1}-\left.\ell^{2} \mathbf{u}^{2}\right|^{2}(t) d(x, y)+v_{u} \int_{\tau}^{t} \int_{D}\left|\nabla\left(\ell^{1} \mathbf{u}^{1}-\ell^{2} \mathbf{u}^{2}\right)\right|^{2} d(x, y) d s \\
& +\varepsilon\left(\int_{D}\left|\ell^{1} q^{1}-\ell^{2} q^{2}\right|^{2}(t) d(x, y)+v_{q} \int_{\tau}^{t} \int_{D}\left|\nabla\left(\ell^{1} q^{1}-\ell^{2} q^{2}\right)\right|^{2} d(x, y) d s\right) \\
& +E \int_{0}^{L}\left\{\left|z^{1}-z^{2}\right|^{2}+f\left|\left(Z^{1}-Z^{2}\right)_{x x}\right|^{2}+a\left|\left(Z^{1}-Z^{2}\right)_{x}\right|^{2}\right. \\
& \left.+b\left|Z^{1}-Z^{2}\right|^{2}\right\}(t) d x \\
& +E \int_{\tau}^{t} \int_{0}^{L}\left\{d\left|\left(z^{1}-z^{2}\right)_{x x}\right|^{2}+e\left|\left(z^{1}-z^{2}\right)_{x t}\right|^{2}+c\left|\left(z^{1}-z^{2}\right)_{x}\right|^{2}\right\} d x d s \\
\leq & \left\|\bar{\eta}^{1}-\bar{\eta}^{2}\right\|_{W^{1, \infty}\left(\mathcal{S}_{\tau, t}\right)}^{2} \omega(\tau, t) \\
& +C \int_{\tau}^{t}\left(\left\|q_{\text {out }}^{1}-q_{\text {out }}^{2}\right\|_{L^{2}\left(S_{\text {out }}\right)}^{2}+\left\|q_{\text {in }}^{1}-q_{\text {in }}^{2}\right\|_{L^{2}\left(S_{\text {in }}\right)}^{2}+\left\|q_{w}^{1}-q_{w}^{2}\right\|_{L^{2}\left(S_{W}\right)}^{2}\right) d s
\end{aligned}
$$

for almost all $t \in[0, T]$, where $\mathcal{S}_{\tau, t}=(0, L) \times(\tau, t), C>0$, and $\omega$ is a positive function $\omega(\tau, t) \rightarrow 0$ as $t \rightarrow \tau$. Moreover, for any $0<q<1$, there is $\iota>0$ such that

$$
\omega(\tau, t) \leq q<1 \quad \text { if } t-\tau \leq \iota
$$


Unfortunately, $\iota$ depends on the regularising parameter $\delta$ in such a way that $\iota=\iota(\delta) \rightarrow 0$ as $\delta \rightarrow 0$.

Proof of Theorem 4.3 Due to tedious calculations, we postpone the proof to the Appendix of this paper.

Consider next the special case $\tau=0, t=T, q_{\text {out }}^{1}=q_{\text {out }}^{2}, q_{\text {in }}^{1}=q_{\mathrm{in}}^{2}, q_{w}^{1}=q_{w}^{2}$, and remember $z=Z_{t}$, then for $\bar{\eta}^{1}, \bar{\eta}^{2} \in \mathcal{K}$, estimate (4.5) gives

$$
\left\|Z^{1}-Z^{2}\right\|_{T}^{2} \leq 2\left\|\bar{\eta}^{1}-\bar{\eta}^{2}\right\|_{C^{1}\left(\bar{S}_{T}\right)}^{2} \omega(0, T) .
$$

Together with the embedding inequality (4.3) this yields continuity of the operator $\mathcal{A}$ : $X \rightarrow X$ on $\mathcal{K}$. Hence, we arrive at the following result.

Theorem 4.4 Suppose that the data satisfy (2.20) and assume $0<\alpha \ll 1$ such small that

$$
4 \alpha<R^{+}+R^{-}<\alpha^{-1}, \text { and let } K=R^{+}-2 \alpha+\left\|\chi^{\prime}\right\|_{\infty} .
$$

In addition, let our regularised parameters be given and satisfy (2.1). Moreover, let

$$
\frac{\mathcal{C}_{e}(T)}{\alpha} M_{q_{b}}(T) \sqrt{1+e^{F T} F T} \leq R^{+}-2 \alpha .
$$

Then $\mathcal{A}$ has a fixed point $\mathcal{A}[\eta]=\eta \in \mathcal{K}$, i.e. there is a solution $(\boldsymbol{u}, q, z)$ to problem (2.9)-(2.17) with

$$
h=h(\eta) \text { and } z=\eta_{t}
$$

on the time interval $[0, T]$. This solution is unique.

Proof Because of Theorem 4.1, Corollary 4.2 and Theorem 4.3, the operator $\mathcal{A}: X \rightarrow$ $X$ is continuous on $\mathcal{K}$ and maps the convex and compact subset $\mathcal{K}$ into itself. Then the existence of $\eta=\mathcal{A}[\eta] \in \mathcal{K}$ follows from the Schauder fixed point theorem. This fixed point is unique due to Theorem 4.3. Indeed, let $\eta^{1} \not \equiv \eta^{2} \in \mathcal{K}$ be two functions with $\eta^{1}=\mathcal{A}\left[\eta^{1}\right]$ and $\eta^{2}=\mathcal{A}\left[\eta^{2}\right]$, and let

$$
\tau:=\sup \left\{s \in[0, T]: \eta^{1}(\cdot, s)=\eta^{2}(\cdot, s)\right\}
$$

Choose now $t \in(\tau, T]$. Since the solution $(\boldsymbol{u}, q, z)=\mathcal{L}(\eta)$ is unique on $[0, \tau] \subset[0, T]$, we have $\left(\boldsymbol{u}^{1}, q^{1}, z^{1}\right)(\cdot, s)=\left(\boldsymbol{u}^{2}, q^{2}, z^{2}\right)(\cdot, s)$ for $s \in[0, \tau]$. Hence, estimate (4.5), the fact that $z^{1}=$ $\eta_{t}^{1}, z^{2}=\eta_{t}^{2}$ together with (4.3) imply

$$
\begin{aligned}
\left\|\eta^{1}-\eta^{2}\right\|_{C^{1}\left(\bar{S}_{\tau, t}\right)}^{2} & \leq\left\|\eta^{1}-\eta^{2}\right\|_{C^{1,1 / 2}\left(\bar{S}_{\tau, t}\right)}^{2} \leq \mathcal{C}_{e}(T-\tau)^{2}\left\|\eta^{1}-\eta^{2}\right\|_{\tau, t}^{2} \\
& \leq 2 \mathcal{C}_{e}(T-\tau)^{2}\left\|\eta^{1}-\eta^{2}\right\|_{C^{1}\left(\bar{S}_{\tau, t}\right)}^{2} \omega(\tau, t),
\end{aligned}
$$

where $\|\xi\|_{\tau, t}$ denotes the norm (4.2) with integration over $[\tau, t]$ instead of $[0, T]$. For $0<$ $t-\tau$ small enough such that $2 \mathcal{C}_{e}(T-\tau)^{2} \omega(\tau, t)<1$, finally, we obtain $\left\|\eta^{1}-\eta^{2}\right\|_{C^{1}\left(\bar{S}_{\tau, t}\right)}=0$, which is a contradiction to the choice of $\tau$. 
Note that our solution $\left(\boldsymbol{u}, q, \eta_{t}\right)$ with $h=h(\eta)$ depends on $\delta, \varepsilon, \kappa$; however, the maximal time interval $[0, T]$ given by (4.7) does not depend on these regularising parameters.

\section{The second a priori estimate}

The aim of this section is to show compactness of our set $\left(\boldsymbol{u}_{\varepsilon, \kappa, \delta}, q_{\varepsilon, \kappa, \delta},\left(\eta_{\varepsilon, \kappa, \delta}\right)_{t}\right)$ with respect to $\delta$ and $\kappa$. Throughout this section let the assumptions of Theorem 4.4 be fulfilled. We start with some obvious estimates concerning the weight $\ell=((h-\delta) y+\delta)(h-\delta)$ based on condition (1.20):

$$
\begin{aligned}
& \alpha^{2}(y+\delta) \leq \ell \leq \alpha^{-2}(y+\delta), \\
& \left|\ell_{x}\right| \leq 2 K \alpha^{-1} \ell, \quad\left|\ell_{t}\right| \leq 2 K \alpha^{-1} \ell, \quad \alpha^{2} \leq \ell_{y} \leq \alpha^{-2}, \\
& |m| \leq K \alpha^{-1}(y+\delta) \leq K \alpha^{-3} \ell .
\end{aligned}
$$

For compactness in time, the following second a priori estimate is essential.

Theorem 5.1 Let $(\boldsymbol{u}, q, z)$ be the solution of free boundary value problem (2.9)-(2.17) given by Theorem 4.4. Then there is a constant $C(\varepsilon)$ independent of $\kappa$ and $\delta$ such that for all $\tau \in[0, T]$ the inequality

$$
\begin{aligned}
& \int_{0}^{T-\tau} \int_{D}|\boldsymbol{u}(t+\tau)-\boldsymbol{u}(t)|^{2}(y+\delta)^{7 / 2} d(x, y) d t \\
& \quad+\varepsilon \int_{0}^{T-\tau} \int_{D}|q(t+\tau)-q(t)|^{2}(y+\delta) d(x, y) d t \leq C(\varepsilon) \tau
\end{aligned}
$$

holds.

Before starting the proof of the theorem, let us prepare some estimations of the nonlinear items.

\section{Lemma 5.2 Let}

$$
\int_{0}^{T} \int_{D}\left(|\nabla \mathbf{u}|^{2}+|\boldsymbol{u}|^{2}\right)(y+\delta) d(x, y) d t+\sup _{t \in[0, T]} \operatorname{ess} \int_{D}|\boldsymbol{u}(x, y, t)|^{2}(y+\delta) d(x, y) \leq C,
$$

then for every fixed $\lambda \geq 5 / 2$, we have

$$
\int_{0}^{T} \int_{D}|\mathbf{u}|^{2}|\nabla \mathbf{u}|(y+\delta)^{\lambda+1} d(x, y) d t \leq C
$$

with generic constants $C$ independent of $\mathbf{u}$ and $\delta$. If, moreover,

$$
\int_{0}^{T} \int_{D}\left|u_{2}\right|^{2}(y+\delta)^{-1} d(x, y) d t \leq C
$$

then

$$
\int_{0}^{T} \int_{D}\left|u_{2}\right||\mathbf{u}|^{2}(y+\delta)^{\lambda} d(x, y) d t \leq C
$$

under the same condition on $\lambda$. 
Proof of Lemma 5.2 Denote $\boldsymbol{w}:=\boldsymbol{u}(y+\delta)^{\beta}$, then $\boldsymbol{w}_{x}=\boldsymbol{u}_{x}(y+\delta)^{\beta}$ and $\boldsymbol{w}_{y}=\boldsymbol{u}_{y}(y+\delta)^{\beta}+\beta \boldsymbol{u}(y+$ $\delta)^{\beta-1}$, hence

$$
|\nabla \boldsymbol{w}|^{2} \leq c\left(|\nabla \boldsymbol{u}|^{2}(y+\delta)^{2 \beta}+|\boldsymbol{u}|^{2}(y+\delta)^{2 \beta-2}\right) .
$$

Then the interpolation inequality (2.27) with $p=4$ implies

$$
\begin{aligned}
\|\boldsymbol{w}\|_{L^{4}\left(0, T ; L^{4}(D)^{2}\right)} \leq & C\|\boldsymbol{w}\|_{L^{\infty}\left(0, T ; L^{2}(D)^{2}\right)}^{1 / 2}\|\nabla \boldsymbol{w}\|_{L^{2}\left(0, T ; L^{2}(D)^{2}\right)}^{1 / 2} \\
\leq & C\left(\operatorname{supess}_{t \in[0, T]} \int_{D}|\boldsymbol{u}(x, y, t)|^{2}(y+\delta)^{2 \beta} d(x, y)\right) \\
& \times\left(\int_{0}^{T} \int_{D}\left(|\nabla \boldsymbol{u}|^{2}(y+\delta)^{2 \beta}+|\boldsymbol{u}|^{2}(y+\delta)^{2 \beta-2}\right) d(x, y) d t\right)^{1 / 4} \\
\leq & C
\end{aligned}
$$

because of (5.5) if $2 \beta>2 \beta-2 \geq 1$, i.e. $\beta \geq 3 / 2$. This yields (5.6) since

$$
\begin{aligned}
& \int_{0}^{T} \int_{D}|\boldsymbol{u}|^{2}|\nabla \mathbf{u}|(y+\delta)^{\lambda+1} d(x, y) d t \\
& \quad=\int_{0}^{T} \int_{D}|\boldsymbol{u}|^{2}\left|(y+\delta)^{\lambda+1 / 2} \nabla \boldsymbol{u}\right|(y+\delta)^{1 / 2} d(x, y) d t \\
& \quad \leq\left(\int_{0}^{T} \int_{D}|\boldsymbol{u}|^{4} \mid(y+\delta)^{2 \lambda+1} d(x, y) d t\right)^{1 / 2}\left(\int_{0}^{T} \int_{D}|\nabla \boldsymbol{u}|^{2}(y+\delta) d(x, y) d t\right)^{1 / 2} \\
& \quad \leq C\|\boldsymbol{w}\|_{L^{4}\left(0, T ; L^{4}(D)\right)}^{2} \leq C
\end{aligned}
$$

due to assumption (5.5) if $\beta=(2 \lambda+1) / 4$, i.e. $\lambda \geq 5 / 2$. Inequality (5.8) follows from Hölder's inequality

$$
\begin{aligned}
\int_{0}^{T} \int_{D}|\boldsymbol{u}|^{2}\left|u_{2}\right|(y+\delta)^{\lambda} d(x, y) d t \leq & \left(\int_{0}^{T} \int_{D}|\boldsymbol{u}|^{4}(y+\delta)^{2 \lambda+1} d(x, y) d t\right)^{1 / 2} \\
& \times\left(\int_{0}^{T} \int_{D}\left|u_{2}\right|^{2}(y+\delta)^{-1} d(x, y) d t\right)^{1 / 2},
\end{aligned}
$$

where the first integral of the product on the right-hand side is bounded owing to (5.9) and the second integral is bounded by our assumption.

Proof of Theorem 5.1 Let $\lambda \geq 0$ be a fixed exponent that will be specified later, let $t, t+\tau \in$ $[0, T]$ be fixed and $\chi_{[t, t+\tau]}$ be the characteristic function of the subinterval $[t, t+\tau] \subset[0, T]$. Replace for a moment the integration variable with respect to time in (2.21) by $s$ instead of $t$ and test it with $\chi_{[t, t+\tau]}(s)(\boldsymbol{w}, p, \zeta)$, where $(\boldsymbol{w}, p, \zeta) \in V$ is independent on $s$. Then the first line of (2.21) passes into

$$
\begin{aligned}
& \rho \int_{D}((\boldsymbol{u} \ell)(x, y, t+\tau)-(\boldsymbol{u} \ell)(x, y, t)) \boldsymbol{w} d(x, y) \\
& \quad+\varepsilon \int_{D}((q \ell)(x, y, t+\tau)-(q \ell)(x, y, t)) p d(x, y)
\end{aligned}
$$


(this follows by approximation from (2.22)). The idea how to derive the estimate of the theorem is now to choose

$$
\begin{aligned}
& \boldsymbol{w}=\partial_{t}^{\tau} \boldsymbol{u}(y+\delta)^{\lambda}:=(\boldsymbol{u}(x, y, t+\tau)-\boldsymbol{u}(x, y, t))(y+\delta)^{\lambda}, \\
& p=\partial_{t}^{\tau} q:=q(x, y, t+\tau)-q(x, y, t), \quad \zeta=\partial_{t}^{\tau} z(1+\delta)^{\lambda}
\end{aligned}
$$

and integrate the resulting relation over $t \in[0, T-\tau]$. This leads to

$$
\begin{aligned}
\rho \int_{0}^{T-\tau} & \int_{D}((\boldsymbol{u} \ell)(x, y, t+\tau)-(\boldsymbol{u} \ell)(x, y, t)) \\
& \times(\boldsymbol{u}(x, y, t+\tau)-\boldsymbol{u}(x, y, t))(y+\delta)^{\lambda} d(x, y) d t \\
& +\varepsilon \int_{0}^{T-\tau} \int_{D}((q \ell)(x, y, t+\tau)-(q \ell)(x, y, t))(q(x, y, t+\tau)-q(x, y, t)) d(x, y) d t \\
& +\int_{0}^{T-\tau} \int_{D}(\ldots \text { further items with } \boldsymbol{u}, q \ldots) d(x, y) d t \\
& +\int_{0}^{T-\tau} \int_{0}^{L}(\ldots \text { further items with } Z, z \ldots) d x d t \\
& +\int_{0}^{T-\tau} \int_{0}^{L} \tau\left(\left[q_{w} h(h-\delta)\right]_{\tau}(x, t) \partial_{t}^{\tau} u_{2}+\kappa\left[u_{2}-z\right]_{\tau}(x, t) \partial_{t}^{\tau}\left(u_{2}-z\right)\right) d x d t \\
= & \int_{0}^{T} \int_{0}^{1} \tau\left(\left[q_{\text {in }} \ell\right]_{\tau} \partial_{t}^{\tau} u_{1}\left(-L^{-}, y, t\right)+\left[q_{\text {out }} \ell\right]_{\tau} \partial_{t}^{\tau} u_{1}\left(L^{+}, y, t\right)\right) d y d t
\end{aligned}
$$

where $[v]_{\tau}=\frac{1}{\tau} \int_{t}^{t+\tau} v(s) d s$ denotes the Steklov average of $v:[0, T] \rightarrow X$ on the interval $[t, t+\tau]$. Note that for any normed space $X$ and $1 \leq p \leq \infty$, the estimate

$$
\left\|[v]_{\tau}\right\|_{L^{p}(0, T-\tau ; X)} \leq\|v\|_{L^{p}(0, T ; X)}
$$

holds. Owing to estimates (5.1) and (5.2), for $\lambda=5 / 2$, the difference of the left-hand side of (5.4) and the first two lines of (5.10) may be estimated by $c \tau$; hence it remains to estimate the items in the other lines of (5.10) also by $c \tau$. Since these are tedious calculations, we restrict ourselves to some exemplary items.

(i) In order to demonstrate the technique, we start with the first item occurring in the third line of (5.10) which is

$$
I_{1}=\left|\frac{\rho}{2} \int_{0}^{T-\tau} \int_{D} \int_{t}^{t+\tau} \boldsymbol{u}(\cdot, s) \cdot(\boldsymbol{u}(\cdot, t+\tau)-\boldsymbol{u}(\cdot, t)) \frac{\partial \ell}{\partial t}(\cdot, s)(y+\delta)^{\lambda} d s d(x, y) d t\right| .
$$

In view of (5.2), (5.1) and $(y+\delta)^{\lambda} \leq c$, we may estimate

$$
\begin{aligned}
I_{1} & \leq c \tau \int_{0}^{T-\tau} \int_{D} \frac{1}{\tau} \int_{t}^{t+\tau}|\boldsymbol{u}(s)| d s(y+\delta)^{1 / 2}|\boldsymbol{u}(t+\tau)-\boldsymbol{u}(t)|(y+\delta)^{1 / 2} d(x, y) d t \\
& \leq c \tau\left\|[|\boldsymbol{u}|]_{\tau}\right\|_{L^{2}\left(0, T-\tau ; L_{2, y+\delta}(D)\right)}\|\boldsymbol{u}(\cdot+\tau)-\boldsymbol{u}\|_{L^{2}\left(0, T-\tau ; L_{2, y+\delta}(D)^{2}\right)} \\
& \leq c \tau\|\boldsymbol{u}\|_{L^{2}\left(0, T ; L_{2, y+\delta}(D)^{2}\right)} \leq c \tau
\end{aligned}
$$

for any $\lambda \geq 0$. In the last steps here, we have used inequality (5.11) and boundedness of the norm due to Theorem 3.1. 
(ii) We proceed with the estimation of the integral arising from the nonlinear items, $c f$. the forth line of (2.21). Recall the definition (2.10) of $\nabla_{h}$, after inserting $\boldsymbol{\psi}=\chi_{[t, t+\tau]}(s)(\boldsymbol{u}(x, y$, $t+\tau)-\boldsymbol{u}(x, y, t))(y+\delta)^{\lambda}$ into $(2.21)$ and integration over $t \in[0, T-\tau]$, the items to be integrated are

$$
\begin{aligned}
\left(\boldsymbol{u} \cdot \nabla_{h}\right) \boldsymbol{u} \cdot \boldsymbol{\psi} \ell= & \left(u_{1} \frac{\partial u_{1}}{\partial x}+m u_{1} \frac{\partial u_{1}}{\partial y}+\frac{1}{h-\delta} u_{2} \frac{\partial u_{1}}{\partial y}\right) \psi_{1} \ell \\
& +\left(u_{1} \frac{\partial u_{2}}{\partial x}+m u_{1} \frac{\partial u_{2}}{\partial y}+\frac{1}{h-\delta} u_{2} \frac{\partial u_{2}}{\partial y}\right) \psi_{2} \ell
\end{aligned}
$$

and

$$
\begin{aligned}
\left(\boldsymbol{u} \cdot \nabla_{h}\right) \boldsymbol{\psi} \cdot \boldsymbol{u} \ell= & \left(u_{1} \frac{\partial \psi_{1}}{\partial x}+m u_{1} \frac{\partial \psi_{1}}{\partial y}+\frac{1}{h-\delta} u_{2} \frac{\partial \psi_{1}}{\partial y}\right) u_{1} \ell \\
& +\left(u_{1} \frac{\partial \psi_{2}}{\partial x}+m u_{1} \frac{\partial \psi_{2}}{\partial y}+\frac{1}{h-\delta} u_{2} \frac{\partial \psi_{2}}{\partial y}\right) u_{2} \ell
\end{aligned}
$$

with

$$
\boldsymbol{u}=\boldsymbol{u}(x, y, s) \quad \text { and } \quad \boldsymbol{\psi}=(\boldsymbol{u}(x, y, t+\tau)-\boldsymbol{u}(x, y, t))(y+\delta)^{\lambda} \quad \text { if } s \in[t, t+\tau]
$$

Observing (5.1), (5.3) and, moreover,

$$
\begin{aligned}
& |\boldsymbol{\psi}| \leq(|\boldsymbol{u}(t+\tau)|+|\boldsymbol{u}(t)|)(y+\delta)^{\lambda}, \\
& \left|\frac{\partial \psi}{\partial y}\right| \leq\left(\left|\frac{\partial \boldsymbol{u}}{\partial y}(t+\tau)\right|+\left|\frac{\partial \boldsymbol{u}}{\partial y}(t)\right|\right)(y+\delta)^{\lambda}+\lambda(|\boldsymbol{u}(t+\tau)|+|\boldsymbol{u}(t)|)(y+\delta)^{\lambda-1},
\end{aligned}
$$

we have to show boundedness of integrals of the form

$$
\begin{aligned}
& \int_{0}^{T-\tau} \int_{D}[|\mathbf{u}|]_{\tau}^{2}|\nabla \mathbf{u}|(y+\delta)^{\tilde{\lambda}+1} d(x, y) d t, \\
& \int_{0}^{T-\tau} \int_{D}|\boldsymbol{u}|[|\mathbf{u}|]_{\tau}[|\nabla \mathbf{u}|]_{\tau}(y+\delta)^{\tilde{\lambda}+1} d(x, y) d t \\
& \int_{0}^{T-\tau} \int_{D}[|\mathbf{u}|]_{\tau}^{2}|\mathbf{u}|(y+\delta)^{\lambda+1} d(x, y) d t, \\
& \int_{0}^{T-\tau} \int_{D}[|\mathbf{u}|]_{\tau}\left[\left|u_{2}\right|\right]_{\tau}|\mathbf{u}|(y+\delta)^{\lambda} d(x, y) d t,
\end{aligned}
$$

where $\tilde{\lambda} \subset\{\lambda, \lambda+1\}$ and $[\cdot]_{\tau}$ is the Steklov average defined after (5.10). Because of $(y+$ $\delta)^{\lambda+1} \leq(1+\alpha)(y+\delta)^{\lambda}$, we can assume $\tilde{\lambda}=\lambda$. Moreover, due to inequality (5.11), we may omit the Steklov averages if the factors under the integrals are separated by Hölder's inequality. Just this is the case in the proof of Lemma 5.2. Hence, we can apply Lemma 5.2 to the integrals (5.14). Since the assumptions of Lemma 5.2 are fulfilled due to Theorem 3.1, the integrals over the items (5.12) and (5.13) are estimated for $\lambda \geq 5 / 2$ by $c \tau$ with a constant $c$ independent of $\kappa, \delta, \varepsilon$.

(iii) The integrals over the items $\mu\left(\left[\nabla_{h} \boldsymbol{u}+\left(\nabla_{h} \boldsymbol{u}\right)^{T}\right]: \nabla_{h} \boldsymbol{\psi}+\frac{2(h-\delta)^{2}}{\ell^{2}} u_{2} \psi_{2}\right) \ell$ and $\varepsilon\left(\nabla_{h} q\right.$. $\nabla_{h}(\omega) \ell$ are estimated in a similar way as in (ii). We consider now

$$
\left(\omega \operatorname{div}_{h} \boldsymbol{u}\right) \ell=\omega\left(\frac{\partial u_{1}}{\partial x}+m \frac{\partial u_{1}}{\partial y}+\frac{1}{h-\delta} \frac{\partial u_{2}}{\partial y}+\frac{h-\delta}{\ell} u_{2}\right) \ell
$$


and

$$
\left(q \operatorname{div}_{h} \boldsymbol{\psi}\right) \ell=q\left(\frac{\partial \psi_{1}}{\partial x}+m \frac{\partial \psi_{1}}{\partial y}+\frac{1}{h-\delta} \frac{\partial \psi_{2}}{\partial y}+\frac{h-\delta}{\ell} \psi_{2}\right) \ell
$$

with

$$
\begin{aligned}
& \boldsymbol{u}=\boldsymbol{u}(x, y, s), \quad q=q(x, y, s) \quad \text { and } \\
& \boldsymbol{\psi}=(\boldsymbol{u}(x, y, t+\tau)-\boldsymbol{u}(x, y, t))(y+\delta)^{\lambda}, \quad \omega=q(x, y, t+\tau)-q(x, y, t)
\end{aligned}
$$

if $s \in[t, t+\tau]$. Hence, in this case, we need boundedness of the integrals

$$
\begin{aligned}
& \int_{0}^{T-\tau} \int_{D}|q|[|\nabla \mathbf{u}|]_{\tau}(y+\delta) d(x, y) d t \\
& \int_{0}^{T-\tau} \int_{D}|q|\left[\left|u_{2}\right|\right]_{\tau} d(x, y) d t \\
& \int_{0}^{T-\tau} \int_{D}[|q|]_{\tau}(|\nabla \mathbf{u}|+|\boldsymbol{u}|)(y+\delta)^{\lambda+1} d(x, y) d t \\
& \int_{0}^{T-\tau} \int_{D}[|q|]_{\tau}\left|u_{2}\right|(y+\delta)^{\lambda} d(x, y) d t .
\end{aligned}
$$

This may be obtained for $\lambda \geq 0$ again by means of Hölder's inequality, (5.11) and the estimates of Theorem 3.1. However, since

$$
\left(\int_{0}^{T} \int_{D}|q|^{2}(y+\delta) d(x, y) d t\right)^{1 / 2} \leq \frac{c}{\sqrt{\varepsilon}}
$$

the integrals over items (5.15) and (5.16) can only be estimated by $c \varepsilon^{-1 / 2} \tau$.

(iv) Since the integrals over the derivatives of $z$ are obviously bounded due to the estimates of Theorem 3.1, finally we have a look at the last item on the left-hand side of (2.21),

$$
\kappa\left(u_{2}-z\right)\left(\psi_{2}-\xi\right)
$$

where $u_{2}=u_{2}(x, 1, s), z=z(x, s), \psi_{2}=\left(u_{2}(x, 1, t+\tau)-u_{2}(x, 1, t)\right)(1+\delta)^{\lambda}, \xi=(z(x, t+\tau)-$ $z(x, t))(1+\delta)^{\lambda}$ if $s \in[t, t+\tau]$. Hence, we obtain

$$
\begin{aligned}
& \tau \int_{0}^{T-\tau} \int_{0}^{L} \kappa\left[\left|u_{2}-z\right|\right]_{\tau}\left|\left(u_{2}(t+\tau)-z(t+\tau)\right)-\left(u_{2}(t)-z(t)\right)\right|(1+\delta)^{\lambda} d x d t \\
& \quad \leq 2 \tau \kappa \int_{0}^{T} \int_{0}^{L}\left|u_{2}-z\right|^{2} d x d t(1+\alpha)^{\lambda} \leq c \tau
\end{aligned}
$$

due to Theorem 3.1 with $c$ independent of $\kappa$. Since the estimation of the right-hand side of (2.21) is obvious, this concludes the proof.

Note that (5.4) depends on $\varepsilon$ also if we test with $\chi_{[t, t+\tau]}(s)(\boldsymbol{w}, 0, \zeta)$ and the corresponding integral in (5.4) disappears. The reason is that it remains to prove boundedness of the integral over item (5.16) which we are not able to estimate independent on $\varepsilon$. Define now

$$
\boldsymbol{w}(x, y, t):=\boldsymbol{u}(x, y, t)(y+\delta)^{7 / 4}
$$


Then from Theorems 3.1 and 5.1 we can derive the following estimates, which yield compactness of $\boldsymbol{w}$ in $L^{2}((0, T) \times D)$.

Lemma 5.3 There are constants $c$ independent of $\delta, \varepsilon, \kappa$ and $\boldsymbol{w}$ such that

$$
\int_{0}^{T-\tau} \int_{D}|\boldsymbol{w}(t+\tau)-\boldsymbol{w}(t)|^{2} d(x, y) d t \leq c \varepsilon^{-1 / 2} \tau
$$

and

$$
\int_{0}^{T} \int_{D} \nabla \boldsymbol{w}: \nabla \boldsymbol{w} d(x, y) d t \leq c
$$

Proof The first estimate (5.20) is equivalent to (5.4) due to the definition of $\boldsymbol{w}$. Hence, it remains to prove (5.21). By means of (5.1), we see that

$$
\begin{aligned}
\boldsymbol{w}_{x}=\boldsymbol{u}_{x}(y+\delta)^{7 / 4} \Rightarrow\left|\boldsymbol{w}_{x}\right|^{2} \leq(1+\alpha)^{5 / 2}\left|\boldsymbol{u}_{x}\right|^{2}(y+\delta) \leq \alpha^{-2}(1+\alpha)^{5 / 2}\left|\boldsymbol{u}_{x}\right|^{2} \ell \quad \text { and } \\
\boldsymbol{w}_{y}=\boldsymbol{u}_{y}(y+\delta)^{7 / 4}+\frac{7}{4} \boldsymbol{u}(y+\delta)^{3 / 4} \\
\Rightarrow \quad\left|\boldsymbol{w}_{y}\right|^{2} \leq 2\left|\boldsymbol{u}_{y}\right|^{2}(y+\delta)(1+\alpha)^{5 / 2}+\frac{49}{8}|\boldsymbol{u}|^{2}(y+\delta)(1+\alpha)^{1 / 2} \\
\leq c\left(\left|\boldsymbol{u}_{y}\right|^{2} \ell+|\boldsymbol{u}|^{2} \ell\right) .
\end{aligned}
$$

Theorem 3.1 then implies (5.21).

To simplify the notation for the limit process $\delta \rightarrow 0$ and $\kappa \rightarrow \infty$ (recall that $\varepsilon$ remains small but fixed), take $k \in \mathbb{N}$, assume

$$
\kappa=k \quad \text { and } \quad \delta=\delta_{k}=k^{-1}
$$

and denote the corresponding solution by $\left(\boldsymbol{u}^{(k)}, q^{(k)}, \eta_{t}^{(k)}\right)$ with $h^{(k)}=h\left(\eta^{(k)}\right)$. This notation applies analogously to all items which depend on $\delta_{k}$ or $\kappa$, e.g. $\boldsymbol{w}^{(k)}=\boldsymbol{u}^{(k)}\left(y+\delta_{k}\right)^{7 / 4}$. Then, as an immediate consequence of Lemma 5.3 and Kolmogorov's compactness theorem [18, Theorem 2.13.1], we obtain the following.

Theorem 5.4 The sequence $\left\{\boldsymbol{w}^{(k)}\right\}_{k=1}^{\infty}$ is precompact in $L^{2}\left(0, T ; L^{2}(D)^{2}\right)$.

Note that the idea of Theorem 5.4 does not apply immediately to $q^{(k)}\left(y+\delta_{k}\right)^{1 / 2}$ since we have no information on boundedness of the gradient of these quantities. An analogue assertion would require to derive the second a priori estimate (5.4) with an weight of higher order in the integral for $q$. Since weak convergence of $q^{(k)}$, however, is enough to go to the limit in Section 6, we forgo these calculations to obtain strong convergence.

We summarise some convergence results concerning $\boldsymbol{u}^{(k)}$ following from Theorems 3.1 and 5.4. For a subsequence $\left(k_{l}\right) \subset \mathbb{N}$ (in the following we write $(k)$ again), it follows

$$
\begin{aligned}
& \left(\ell^{(k)}\right)^{1 / 2} \boldsymbol{u}^{(k)} \stackrel{*}{\rightarrow} h y^{1 / 2} \boldsymbol{u} \quad \text { in } L^{\infty}\left(0, T ; L^{2}(D)^{2}\right), \\
& \left(\ell^{(k)}\right)^{1 / 2} \boldsymbol{u}^{(k)} \rightarrow h y^{1 / 2} \boldsymbol{u} \quad \text { in } L^{2}\left(0, T ; L^{2}(D)^{2}\right),
\end{aligned}
$$




$$
\begin{aligned}
& \left(h-\delta_{k}\right)\left(\ell^{(k)}\right)^{-1 / 2} u_{2}^{(k)}-y^{-1 / 2} u_{2} \quad \text { in } L^{2}\left(0, T ; L^{2}(D)\right), \\
& \left(\ell^{(k)}\right)^{1 / 2} \nabla \boldsymbol{u}^{(k)} \rightarrow h y^{1 / 2} \nabla \boldsymbol{u} \quad \text { in } L^{2}\left(0, T ; L^{2}(D)^{2}\right), \\
& \boldsymbol{w}^{(k)} \rightarrow \boldsymbol{w} \quad \text { in } L^{2}\left(0, T ; L^{2}(D)^{2}\right) .
\end{aligned}
$$

We still have to formulate the convergence properties for the other components of our solution.

Theorem 5.5 For an infinite subset of $\{k: k \in \mathbb{N}\}$, we have

$$
\begin{aligned}
& \left(\varepsilon \ell^{(k)}\right)^{1 / 2} q^{(k)} \stackrel{*}{\rightarrow} \varepsilon^{1 / 2} h y^{1 / 2} q \quad \text { in } L^{\infty}\left(0, T ; L^{2}(D)\right), \\
& \left(\varepsilon \ell^{(k)}\right)^{1 / 2} q^{(k)} \rightarrow \varepsilon^{1 / 2} h y^{1 / 2} q \quad \text { in } L^{2}\left(0, T ; L^{2}(D)\right), \\
& \left(\varepsilon \ell^{(k)}\right)^{1 / 2} \nabla q^{(k)} \rightarrow \varepsilon^{1 / 2} h y^{1 / 2} \nabla q \quad \text { in } L^{2}\left(0, T ; L^{2}(D)\right), \\
& \eta^{(k)} \rightarrow \eta \quad \text { in } H_{0-}^{1}\left(0, T ; H_{0}^{2}(0, L)\right) \cap H_{0-}^{2}\left(0, T ; H_{0}^{1}(0, L)\right), \\
& \eta^{(k)} \rightarrow \eta \quad \text { in } C^{1}([0, T] \times[0, L]) .
\end{aligned}
$$

Note that $Z^{(k)}=\eta^{(k)}, Z:=\eta, z^{(k)}=\left(\eta^{(k)}\right)_{t}, z:=\eta_{t}$, hence we have corresponding convergence properties for $Z^{(k)}$ and $z^{(k)}$, respectively.

Proof Relations (5.28)-(5.30) again are a consequence of the estimates of Theorem 3.1. Moreover, due to the a priori estimate (3.1), $\left\{\eta^{(k)}\right\}$ has a bounded norm (4.2) in $H_{0-}^{1}(0, T$; $\left.H_{0}^{2}(0, L)\right) \cap H_{0-}^{2}\left(0, T ; H_{0}^{1}(0, L)\right)$, hence this implies (5.31). Equation (5.32) then follows from the compact embedding (4.3).

An immediate but important consequence of (5.32) is the uniform convergence of

$$
h^{(k)}=h\left(\eta^{(k)}\right) \rightarrow h:=h(\eta) \quad \text { in } C^{1}([0, T] \times[0, L])
$$

and

$$
\ell^{(k)} \rightarrow h^{2} y \quad \text { in } C^{1}([0, T] \times[0, L])
$$

This belatedly justifies the coefficient $h y^{1 / 2}$ in the limits (5.23)-(5.30). Moreover, we conclude $\boldsymbol{w}=y^{7 / 4} \boldsymbol{u}$ because of

$$
\begin{aligned}
\int_{0}^{T} & \int_{D} \boldsymbol{w}^{(k)} \cdot \boldsymbol{\psi} d(x, y) d t \\
& =\int_{0}^{T} \int_{D} \boldsymbol{u}^{(k)}\left(\ell^{(k)}\right)^{1 / 2} \underbrace{\left(y+\delta_{k}\right)^{7 / 4}\left(\ell^{(k)}\right)^{-1 / 2}}_{=: \mu_{k}} \boldsymbol{\psi} d(x, y) d t \\
& \rightarrow \int_{0}^{T} \int_{D} \boldsymbol{u} h y^{1 / 2}\left(h^{-1} y^{5 / 4}\right) \boldsymbol{\psi} d(x, y) d t \\
& =\int_{0}^{T} \int_{D} y^{7 / 4} \boldsymbol{u} \cdot \boldsymbol{\psi} d(x, y) d t
\end{aligned}
$$


for all $\psi \in L^{2}\left(0, T ; L^{2}(D)^{2}\right)$ and the uniform convergence $\mu_{k} \rightarrow \frac{y^{7 / 4}}{h y^{1 / 2}}$ in $(0, T) \times D$. Indeed, the following holds:

$$
\begin{aligned}
\left|\mu_{k}-\frac{y^{5 / 4}}{h}\right| & \leq \frac{\left|\left(y+\delta_{k}\right)^{7 / 4}-y^{7 / 4}\right|}{\left(l^{(k)}\right)^{1 / 2}}+\frac{\left|h y^{1 / 2}-\left(l^{(k)}\right)^{1 / 2}\right|}{h\left(l^{(k)}\right)^{1 / 2}} y^{5 / 4} \\
& \leq \frac{c \delta_{k}}{\alpha \delta_{k}^{1 / 2}}+\frac{\left|h^{2} y-l^{(k)}\right|^{1 / 2}}{h \alpha y^{1 / 2}} y^{5 / 4} \rightarrow 0 \quad \text { uniformly as } \delta_{k} \rightarrow 0,
\end{aligned}
$$

where we have used $l^{(k)} \geq \alpha^{2} \delta_{k}$ and $l^{(k)} \geq \alpha^{2} y(c f$. (5.1)) and (5.34).

Instead of (5.27), however, we obtain the unweighted strong convergence of $\boldsymbol{u}^{(k)}$ in $L^{2}\left(0, T ; L^{2}(D)^{2}\right)$. To prove this, we start with the following.

Lemma 5.6 Set $\boldsymbol{v}=\boldsymbol{u}^{(k)}-\boldsymbol{u}$. Then, for each sufficiently small $0<\iota \ll 1$ and arbitrary $\beta>0$, we have the estimate

$$
\begin{aligned}
& \int_{0}^{T} \int_{D}|\mathbf{v}|^{2} y^{\beta+\iota-1 / 3} d(x, y) d t \\
& \quad \leq C_{\beta, \iota}\left(\int_{0}^{T} \int_{D}|\nabla \mathbf{v}|^{2} y d(x, y) d t\right)^{\theta}\left(\int_{0}^{T} \int_{D}|\mathbf{v}|^{2} y^{\beta+1} d(x, y) d t\right)^{1-\theta}
\end{aligned}
$$

the constant $C_{\beta, \iota}$ depending on $\beta, \iota$ in such a fashion that $C_{\beta, \iota} \rightarrow \infty$ if $\beta \rightarrow 0$ or $\iota \rightarrow 0$, and $\theta=1-\iota / 2$. Note that $C_{\beta, \imath}$ does not depend on $k$.

Proof We compute

$$
\begin{aligned}
\int_{D}|\boldsymbol{v}|^{2} y^{\lambda} d(x, y) & \leq\left(\int_{D}|\boldsymbol{v}|^{2 p} y^{(\lambda+\gamma) p} d(x, y)\right)^{1 / p}\left(\int_{D} y^{-\gamma p^{\prime}} d(x, y)\right)^{1 / p^{\prime}} \\
& =\left(\frac{L^{+}+L^{-}}{1-\gamma p^{\prime}}\right)^{1 / p^{\prime}}\left(\int_{D}|\boldsymbol{w}|^{2 p} y d(x, y)\right)^{1 / p}
\end{aligned}
$$

assuming $\lambda \in \mathbb{R}, 0<\gamma<\frac{1}{p^{\prime}}=1-\frac{1}{p}$ and $\boldsymbol{w}=\boldsymbol{v} y^{\beta / 2}$ with $\beta=\lambda+\gamma-1 / p$. We next apply the interpolation inequality (2.28) to deduce

$$
\left(\int_{D}|\boldsymbol{w}|^{2 p} y d(x, y)\right)^{1 / p} \leq C^{2}\|\nabla \boldsymbol{w}\|_{L_{y}^{2}(D)^{2}}^{2 \theta}\|\boldsymbol{w}\|_{L_{y}^{2}(D)^{2}}^{2(1-\theta)} \text { for } \frac{3}{2}\left(1-\frac{1}{p}\right)=\theta<1 .
$$

Since $\nabla \boldsymbol{w}=\nabla \boldsymbol{v} y^{\beta / 2}+\frac{\beta}{2} \boldsymbol{v} y^{-1+\beta / 2},|\nabla \boldsymbol{w}|^{2} y \leq c\left(|\nabla \boldsymbol{v}|^{2} y^{\beta+1}+|\boldsymbol{v}|^{2} y^{\beta-1}\right)$. But then, assuming $\beta>0$ and due to (3.8) and (3.9), we arrive at $\|\nabla \boldsymbol{w}\|_{L_{y}^{2}(D)^{2}} \leq \frac{C_{1}}{\beta}\|\nabla \boldsymbol{v}\|_{L_{y}^{2}(D)^{2}}$, and, finally,

$$
\begin{aligned}
& \int_{D}|\boldsymbol{v}|^{2} y^{\lambda} d(x, y) \\
& \quad \leq\left(\frac{1}{p^{\prime}} \frac{L^{+}+L^{-}}{1-\gamma-1 / p}\right)^{1 / p^{\prime}}\left(\frac{C_{1}}{\beta}\|\nabla \boldsymbol{v}\|_{L_{y}^{2}(D)^{2}}\right)^{2 \theta}\left(\int_{D}|\boldsymbol{v}|^{2} y^{\beta+1} d(x, y)\right)^{1-\theta} .
\end{aligned}
$$

Now, let us summarise some relations between our parameters

$$
0<\gamma<1-\frac{1}{p}<\frac{2}{3} \text { and } \beta=\lambda+\gamma-\frac{1}{p}
$$


and set

$$
\gamma+\varepsilon_{1}=1-\frac{1}{p}+\varepsilon_{2}=\frac{2}{3} \quad \text { for } \varepsilon_{1}>\varepsilon_{2}>0
$$

If we take $\varepsilon_{1}=2 \varepsilon_{2}$ and $\iota=3 \varepsilon_{2}$, then $\lambda=\beta+\iota-1 / 3$ and (5.35) follows after integration over $(0, T)$.

By means of the above lemma, we may sharpen the convergence assertion (5.24).

Corollary 5.7 For every fixed $\lambda>-\frac{1}{3}$, we have the convergence property

$$
\int_{0}^{T} \int_{D}\left|\boldsymbol{u}^{(k)}-\boldsymbol{u}\right|^{2} y^{\lambda} d(x, y) d t \rightarrow 0 \quad \text { as } k \rightarrow \infty
$$

Especially for $\lambda=0$, the following holds:

$$
\boldsymbol{u}^{(k)} \rightarrow \boldsymbol{u} \quad \text { in } L^{2}\left(0, T ; L^{2}(D)^{2}\right)
$$

Proof We proceed in an iterative way.

(1) From (5.27) we have

$$
\int_{0}^{T} \int_{D}\left|\boldsymbol{u}^{(k)}-\boldsymbol{u}\right|^{2} y^{1+5 / 2} d(x, y) d t \rightarrow 0 \quad \text { as } k \rightarrow \infty,
$$

hence by Lemma 5.6 for $\beta_{1}=5 / 2$ also

$$
\int_{0}^{T} \int_{D}\left|\boldsymbol{u}^{(k)}-\boldsymbol{u}\right|^{2} y^{1+t+7 / 6} d(x, y) d t \rightarrow 0 \quad \text { as } k \rightarrow \infty .
$$

(2) We apply again Lemma 5.6, but now for $\beta_{2}=\iota+7 / 6$, and we obtain

$$
\int_{0}^{T} \int_{D}\left|\boldsymbol{u}^{(k)}-\boldsymbol{u}\right|^{2} y^{2 \ell+5 / 6} d(x, y) d t \rightarrow 0 \quad \text { as } k \rightarrow \infty
$$

As $\iota$ can be taken arbitrarily small, (5.36) holds for any $\lambda>5 / 6$.

(3) Fix $\lambda>-1 / 3$. Now choose positive $\beta$ and $\iota$ such that $\lambda=\beta+\iota-1 / 3$. The conclusion of item (2) and estimate (5.35) then yield

$$
\int_{0}^{T} \int_{D}\left|\boldsymbol{u}^{(k)}-\boldsymbol{u}\right|^{2} y^{\lambda} d(x, y) d t \leq \mathrm{const} . \int_{0}^{T} \int_{D}\left|\boldsymbol{u}^{(k)}-\boldsymbol{u}\right|^{2} y^{\beta+1} d(x, y) d t \rightarrow 0
$$

as $k \rightarrow \infty$ because of $\beta+1>5 / 6$. This is the assertion of the corollary.

Furthermore, since the integral $\int_{0}^{T} \int_{D}\left|u_{2}\right|^{2} y^{-1} d(x, y) d t$ is finite, we obtain from [19, Remark 7.8 or Proposition 9.10] the following.

Corollary 5.8 $u_{2}=0$ on $\left\{(x, 0, t):-L^{-} \leq x \leq L^{+}, 0 \leq t \leq T\right\}$ in the sense of traces. 


\section{The original problem}

In this section we want to show that our original fluid-structure interaction problem (1.1)(1.14) with small but fixed $\varepsilon$ has a weak solution. To start with the definition of a weak solution again, we use a transformation of the time dependent domain $\Omega_{h}$ defined by (1.3) on our fixed domain $D$, now given by (1.16) instead of (2.7). Note that the $h$ given by (1.4) now will be defined using the limit function $\eta$ given by Theorem 5.5 and thus, contrary to transformation (2.7), it is a fixed known function in the following. Moreover, transformation (1.16) is smooth since $h \in C^{1,1 / 2}([0, T] \times[0, L])$.

Definition 6.1 We call $\left(v_{x}, v_{r}, p, \eta\right)$ a weak solution of the original problem (1.1)-(1.14) if there is an $\eta \in W^{1, \infty}((0, L) \times(0, T))$ fulfilling

$$
\eta_{t}(x, t)=v_{r}(x, h(x, t), t)
$$

where $h$ is defined by $(1.4)$ and such that the triple $(\boldsymbol{u}, q, \eta)$ given by $(1.16)$ has the following properties:

(1) $\boldsymbol{u} \in L^{\infty}\left(0, T ; L_{y}^{2}(D)^{2}\right) \cap L^{2}\left(0, T ; \boldsymbol{V}_{y}\right), q \in L^{\infty}\left(0, T ; L_{y}^{2}(D)\right) \cap L^{2}\left(0, T ; H_{y}^{1}(D)\right), \eta \in H_{0-}^{1}(0$, $\left.T ; H_{0}^{2}(0, L)\right) \cap H_{0-}^{2}\left(0, T ; H_{0}^{1}(0, L)\right)$.

(2) $(\boldsymbol{u}, q, \eta)$ satisfies the following relation:

$$
\begin{aligned}
-\rho & \int_{0}^{T} \int_{D} \boldsymbol{u} \frac{\partial \boldsymbol{\psi}}{\partial t} h^{2} y d(x, y) d t-\varepsilon \int_{0}^{T} \int_{D} q \frac{\partial \omega}{\partial t} h^{2} y d(x, y) d t \\
& -\frac{\rho}{2} \int_{0}^{T} \int_{D}\left\{(\boldsymbol{u} \cdot \boldsymbol{\psi}) 2 h \frac{\partial h}{\partial t} y+\left[\frac{\partial \boldsymbol{u}}{\partial y} \cdot \boldsymbol{\psi}-\boldsymbol{u} \cdot \frac{\partial \boldsymbol{\psi}}{\partial y}\right] h \frac{\partial h}{\partial t} y^{2}\right\} d(x, y) d t \\
& -\frac{\varepsilon}{2} \int_{0}^{T} \int_{D}\left\{q \omega 2 h \frac{\partial h}{\partial t} y+\left[\frac{\partial q}{\partial y} \omega-q \frac{\partial \omega}{\partial y}\right] h \frac{\partial h}{\partial t} y^{2}\right\} d(x, y) d t \\
& +\int_{0}^{T} \int_{D}\left\{\frac{\rho}{2}\left[\left(\left(\boldsymbol{u} \cdot \nabla_{h}\right) \mathbf{u}\right) \cdot \boldsymbol{\psi}-\left(\left(\boldsymbol{u} \cdot \nabla_{h}\right) \boldsymbol{\psi}\right) \cdot \boldsymbol{u}\right]\right. \\
& \left.+\mu\left(\left[\nabla_{h} \mathbf{u}+\left(\nabla_{h} \boldsymbol{u}\right)^{T}\right]: \nabla_{h} \boldsymbol{\psi}+\frac{2}{(h y)^{2}} u_{2} \psi_{2}\right)\right\} h^{2} y d(x, y) d t \\
& +\int_{0}^{T} \int_{D}\left\{\varepsilon\left(\nabla_{h} q \cdot \nabla_{h} \omega\right)+\left(\omega \operatorname{div} v_{h} \boldsymbol{u}-q \operatorname{div}_{h} \boldsymbol{\psi}\right)\right\} h^{2} y d(x, y) d t \\
& +E \int_{0}^{T} \int_{0}^{L}\left\{-\eta_{t} \xi_{t}+d \eta_{t x x} \xi_{x x}+e \eta_{t x t} \xi_{x t}+f \eta_{x x} \xi_{x x}\right. \\
& \left.+c \eta_{x t} \xi_{x}+a \eta_{x} \xi_{x}+b \eta \xi\right\} d x d t \\
& +\int_{0}^{T} \int_{0}^{L} q_{w} \psi_{2} h^{2} d x d t \\
= & \int_{0}^{T} \int_{0}^{1}\left(q_{\text {in }}\left(\psi_{1} h^{2}\right)\left(-L^{-}, y, t\right)+q_{\text {out }}\left(\psi_{1} h^{2}\right)\left(L^{+}, y, t\right)\right) y d y d t
\end{aligned}
$$

for every $(\boldsymbol{\psi}, \omega, \xi) \in L^{2}\left(0, T ; V_{y}\right), \boldsymbol{\psi} \in L^{5}\left(0, T ; H_{y}^{1,5}(D)^{2}\right) \cap H^{1,1}\left(0, T ; L_{y}^{2}(D)^{2}\right), \omega \in H^{1,1}(0, T$; $\left.L_{y}^{2}(D)\right), \xi \in H^{1,1}\left(0, T ; H_{0}^{1}(0, L)\right)$ with $(\boldsymbol{\psi}, \omega, \xi)(\cdot, T)=\mathbf{0}$ and $\psi_{2}=\xi$ a.e. on $(0, T) \times\left(S_{w} \backslash \Gamma\right)$.

Note that condition (6.1) and $\eta_{t}=h_{t}$ on $(0, T) \times(0, L)$ imply that the artificial item $-\frac{\rho}{2} v_{r}\left(v_{r}-h_{t}\right)$ in (2.5) disappears. 
We will show that the limit $(\boldsymbol{u}, q, \eta)$ given by (5.23)-(5.32) provides a solution in the sense of Definition 6.1. First we check (6.1). From the first a priori estimate (3.1) with $\kappa=k$, especially, we obtain the inequality

$$
\int_{0}^{T} \int_{0}^{L}\left|u_{2}^{(k)}(x, 1, t)-\eta_{t}^{(k)}(x, t)\right|^{2} d x d t \leq \frac{C}{k}
$$

with some constant $C$ independent of $k$. The interpolation inequality

$$
\|\varphi\|_{L^{2}\left(0, T ; L^{r}\left(S_{w}\right)\right)} \leq C\|\varphi\|_{L^{2}\left(0, T ; H^{1}(\widetilde{D})\right)}^{\theta}\|\varphi\|_{L^{2}\left(0, T ; L^{2}(\widetilde{D})\right)}^{1-\theta}
$$

$1<r<\infty, 1-1 / r \leq \theta \leq 1$ (cf. [16, Chapter II, formula (2.21)]) applied to $\varphi=u_{2}^{(k)}-u_{2}$ in $\widetilde{D}=\left(-L^{-}, L^{+}\right) \times(\alpha, 1)$ yields the strong convergence

$$
u_{2}^{(k)} \rightarrow u_{2} \quad \text { in } L^{2}\left(0, T ; L^{r}\left(S_{w}\right)\right)
$$

as $k \rightarrow \infty$ as a consequence of (5.24), (5.26) and (5.37). Together with the uniform convergence (5.32) of $\left(\eta^{(k)}\right)_{t}$, this convergence (6.4) and (6.3) for $k \rightarrow \infty$ yield $u_{2}=\eta_{t}$ a.e. on $(0, T) \times\left(S_{w} \backslash \Gamma\right)$, which is (6.1). Moreover, $u_{2}=0$ on $S_{c}$ due to Corollary 5.8. The other homogeneous boundary conditions for $\boldsymbol{u}$ included in the definition of $\boldsymbol{V}_{y}$ and for $\eta$ are fulfilled because of the convergence properties (5.23)-(5.32) since $\boldsymbol{u}^{(k)}$ and $\eta^{(k)}$, respectively, satisfy it. By the same reason $(\boldsymbol{u}, q, \eta)$ belongs to the function spaces given in item (1) of Definition 6.1.

It remains to prove relation (6.2). To this end, choose a smooth test function $(\psi, \omega, \xi)$ belonging to the spaces given in item (2) of Definition 6.1 and consider relation (2.21) from Definition 2.1 for our sequence $\left(\boldsymbol{u}^{(k)}, q^{(k)}, \eta^{(k)}\right)$,

$$
\begin{aligned}
-\int_{0}^{T} & \int_{D}\left(\rho \boldsymbol{u}^{(k)} \ell^{(k)} \frac{\partial \boldsymbol{\psi}}{\partial t}+\varepsilon q^{(k)} \ell^{(k)} \frac{\partial \omega}{\partial t}\right) d(x, y) d t \\
& -\frac{\rho}{2} \int_{0}^{T} \int_{D}\left\{\left(\boldsymbol{u}^{(k)} \cdot \boldsymbol{\psi}\right) \frac{\partial \ell^{(k)}}{\partial t}\right. \\
& \left.+\left[\frac{\partial \boldsymbol{u}^{(k)}}{\partial y} \cdot \boldsymbol{\psi}-\boldsymbol{u}^{(k)} \cdot \frac{\partial \boldsymbol{\psi}}{\partial y}\right]\left[\left(h^{(k)}-\frac{1}{k}\right) y+\frac{1}{k}\right] \frac{\partial h^{(k)}}{\partial t} y\right\} d(x, y) d t \\
& -\frac{\varepsilon}{2} \int_{0}^{T} \int_{D}\left\{q^{(k)} \omega \frac{\partial \ell^{(k)}}{\partial t}\right. \\
& \left.+\left[\frac{\partial q^{(k)}}{\partial y} \omega-q^{(k)} \frac{\partial \omega}{\partial y}\right]\left[\left(h^{(k)}-\frac{1}{k}\right) y+\frac{1}{k}\right] \frac{\partial h^{(k)}}{\partial t} y\right\} d(x, y) d t \\
& +\int_{0}^{T} \int_{D}\left\{\frac{\rho}{2}\left[\left(\left(\boldsymbol{u}^{(k)} \cdot \nabla_{h^{(k)}}\right) \boldsymbol{u}^{(k)}\right) \cdot \boldsymbol{\psi}-\left(\left(\boldsymbol{u}^{(k)} \cdot \nabla_{h^{(k)}}\right) \boldsymbol{\psi}\right) \cdot \boldsymbol{u}^{(k)}\right] \ell^{(k)}\right. \\
& +\mu\left(\left[\nabla_{h^{(k)}} \boldsymbol{u}^{(k)}+\left(\nabla_{h^{(k)}} \boldsymbol{u}^{(k)}\right)^{T}\right]: \nabla_{h^{(k)}} \boldsymbol{\psi}\right. \\
& \left.+\frac{2\left(h^{(k)}-k^{-1}\right)^{2}}{\left(\ell^{(k)}\right)^{2}} u_{2}^{(k)} \psi_{2}\right) \ell^{(k)}+\varepsilon\left(\nabla_{h^{(k)}} q^{(k)} \cdot \nabla_{h^{(k)}} \omega\right) \ell^{(k)} \\
& \left.+\left(\omega \operatorname{div}_{h^{(k)}} \boldsymbol{u}^{(k)}-q^{(k)} \operatorname{div}_{h^{(k)}} \boldsymbol{\psi}\right) \ell^{(k)}\right\} d(x, y) d t
\end{aligned}
$$




$$
\begin{aligned}
& +E \int_{0}^{T} \int_{0}^{L}\left\{-\eta_{t}^{(k)} \xi_{t}+d \eta_{t x x}^{(k)} \xi_{x x}+e \eta_{t x t}^{(k)} \xi_{x t}+f \eta_{x x}^{(k)} \xi_{x x}\right. \\
& \left.+c \eta_{x t}^{(k)} \xi_{x}+a \eta_{x}^{(k)} \xi_{x}+b \eta^{(k)} \xi\right\} d x d t \\
& +k \int_{0}^{T} \int_{0}^{L}\left(u_{2}^{(k)}-\eta_{t}^{(k)}\right)\left(\psi_{2}(x, 1, t)-\xi(x, t)\right) d x d t \\
& +\int_{0}^{T} \int_{0}^{L} q_{w} \psi_{2} h^{(k)}\left(h^{(k)}-\frac{1}{k}\right) d x d t \\
& =\int_{0}^{T} \int_{0}^{1}\left(\left(q_{\text {in }} \psi_{1} \ell^{(k)}\right)\left(-L^{-}, y, t\right)+\left(q_{\text {out }} \psi_{1} \ell^{(k)}\right)\left(L^{+}, y, t\right)\right) d y d t
\end{aligned}
$$

We want to show that (6.5) tends to (6.2) if $k \rightarrow \infty$ for the corresponding subsequence such that (5.23)-(5.32) hold. It is obvious from (5.24), (5.29), (5.37) and (5.34) that the first two lines of (6.5) tend to the first three items of (6.2). We demonstrate the limit of the first item on the third line. Denote $\boldsymbol{\Psi}^{(k)}=\boldsymbol{\psi}\left[\left(h^{(k)}-1 / k\right) y+1 / k\right]\left(\partial h^{(k)} / \partial t\right)$ and $\boldsymbol{\Psi}=\boldsymbol{\psi} h h_{t} y$, then we have to evaluate

$$
\begin{aligned}
\int_{0}^{T} & \int_{D}\left(\nabla \boldsymbol{u}^{(k)} \cdot \boldsymbol{\Psi}^{(k)} y-\nabla \boldsymbol{u} \cdot \boldsymbol{\Psi} y\right) d(x, y) d t \\
= & \int_{0}^{T} \int_{D} y^{1 / 2} \nabla \boldsymbol{u}^{(k)} \cdot \boldsymbol{\Psi}^{(k)}\left(y^{1 / 2}-\left(\ell^{(k)}\right)^{1 / 2} / h^{(k)}\right) d(x, y) d t \\
& \quad+\int_{0}^{T} \int_{D}\left(\ell^{(k)}\right)^{1 / 2} \nabla \boldsymbol{u}^{(k)} \cdot\left(\boldsymbol{\Psi}^{(k)} y^{1 / 2} / h^{(k)}-\boldsymbol{\Psi} y^{1 / 2} / h\right) d(x, y) d t \\
& +\int_{0}^{T} \int_{D}\left(\left(\ell^{(k)}\right)^{1 / 2} \nabla \boldsymbol{u}^{(k)}-h y^{1 / 2} \nabla \mathbf{u}\right) \cdot \boldsymbol{\Psi} y^{1 / 2} / h d(x, y) d t=I_{1}+I_{2}+I_{3} .
\end{aligned}
$$

The first two integrals $I_{1}+I_{2}$ tend to zero since

$$
\alpha y^{1 / 2}\left|\nabla \boldsymbol{u}^{(k)}\right| \leq\left(\ell^{(k)}\right)^{1 / 2}\left|\nabla \boldsymbol{u}^{(k)}\right|
$$

(cf. (5.1)) is bounded in $L^{2}\left(0, T ; L^{2}(D)\right)$ and the remaining factors converge uniformly to zero due to (5.33), (5.34). The integral $I_{3}$ converges to zero because of the weak convergence (5.26).

Similar calculations yield the convergence of the nonlinear item on the sixth line and the elliptic item on the seventh line of (6.5), respectively, to the corresponding items of the relation (6.2) as well as the convergence of the second item on line 4 including the pressure $q^{(k)}$. For the nonlinear items, it is sufficient to have the strong convergence (5.37) of $\boldsymbol{u}^{(k)}$ and the weak convergence (5.26) for the gradient both in Hilbert spaces if $\boldsymbol{\psi}$ is chosen smooth and therefore pointwise bounded together with its derivatives. Thereby, it is important that $h^{(k)}$ and its derivatives converge uniformly in $[0, T] \times[0, L], c f .(5.33)$.

Next the convergence

$$
\int_{0}^{T} \int_{D} q^{(k)} \omega \frac{\partial \ell^{(k)}}{\partial t} d(x, y) d t \rightarrow \int_{0}^{T} \int_{D} q \omega 2 h \frac{\partial h}{\partial t} y d(x, y) d t
$$


follows since (3.10) for $\lambda=0$ implies the weak convergence of $q^{(k)}$ in the unweighted space $L^{2}\left(0, T ; L^{2}(D)\right)$. Finally, it remains to establish the convergence

$$
\begin{aligned}
\int_{0}^{T} & \int_{D} \frac{2\left(h^{(k)}-k^{-1}\right)^{2}}{\left(\ell^{(k)}\right)^{2}} u_{2}^{(k)} \psi_{2} \ell^{(k)} d(x, y) d t \\
= & \int_{0}^{T} \int_{D} 2 \frac{h^{(k)}-\delta_{k}}{\left(\ell^{(k)}\right)^{1 / 2}} u_{2}^{(k)} \frac{h^{(k)}-\delta_{k}}{\left(\ell^{(k)}\right)^{1 / 2}} \psi_{2} d(x, y) d t \\
& \rightarrow \int_{0}^{T} \int_{D} \frac{2}{y} u_{2} \psi_{2} d(x, y) d t .
\end{aligned}
$$

If $\boldsymbol{\psi}$ is smooth, say $\boldsymbol{\psi} \in C^{1}([0, T] \times \bar{D})^{2}$ with $\psi_{2}(x, 0, t)=0$, we have $\left|\psi_{2}\right| \leq C y$. Hence (6.6) follows from the weak convergence (5.25) and the uniform convergence

$$
\begin{aligned}
\left|\frac{h^{(k)}-\delta_{k}}{\left(\ell^{(k)}\right)^{1 / 2}}-\frac{1}{y^{1 / 2}}\right|\left|\psi_{2}\right| & =\frac{\left|\left(h^{(k)}-\delta_{k}\right) y^{1 / 2}-\left(\ell^{(k)}\right)^{1 / 2}\right|}{\left(\ell^{(k)}\right)^{1 / 2} y^{1 / 2}}\left|\psi_{2}\right| \\
& \leq \frac{\left|\left(h^{(k)}-\delta_{k}\right) y^{1 / 2}-\left(\ell^{(k)}\right)^{1 / 2}\right|}{\alpha y} C y \rightarrow 0
\end{aligned}
$$

due to (5.1) and (5.34). The convergence of the remaining items of (6.5) to the corresponding items of relation (6.2) is obvious.

Now we are ready to formulate our main existence result.

Theorem 6.1 Suppose that the data satisfy (2.20) and assume $0<\alpha \ll 1$ such small that $4 \alpha<R^{+}+R^{-}<\alpha^{-1}$, and let $K=R^{+}-2 \alpha+\left\|\chi^{\prime}\right\|_{\infty}$.

Then there is $T>0$ and a weak solution $\left(v_{x}, v_{r}, p, \eta\right)$ of the original problem (1.1)-(1.14) on $[0, T]$ in the sense of Definition 6.1 where the moving boundary $h$ is defined by (1.4) in terms of the component $\eta$ and $h$ fulfils estimates (1.20).

Proof From the above considerations, it is clear that the limit $(\boldsymbol{u}, q, \eta)$ defined in Section 5 fulfils (6.1) and relation (6.2) for $\xi \in L^{2}\left(0, T ; H_{0}^{2}\left(-L^{-}, L^{+}\right)\right) \cap H^{1,1}\left(0, T ; H_{0}^{1}(0, L)\right)$ and smooth $(\psi, q) \in C^{1}([0, T] \times \bar{D})^{3}$ satisfying the corresponding boundary conditions. Now we make some remarks concerning the density argument. Owing to [19, Theorem 7.2], the embedding $C^{1}(\bar{D}) \subset H_{y}^{1, r}(D)$ is dense for every $r \geq 1$. Moreover, let $C_{c}^{1}(\bar{D})=\left\{\psi \in C^{1}(\bar{D})\right.$ : supp $\psi \cap S_{c}=\emptyset$. Then $C_{c}^{1}(\bar{D})$ is dense in the set of all functions $u$ with $y^{-1 / 2} u \in L^{2}(D)$, cf. [19, Remark 7.8 and Theorem 11.11]. Hence, the set

$$
\left\{(\boldsymbol{\psi}, q) \in C^{1}(\bar{D})^{3}: \operatorname{supp} \psi_{1} \cap S_{w}=\emptyset, \operatorname{supp} \psi_{2} \cap S_{\text {in }} \cap S_{\text {out }} \cap S_{c}=\emptyset\right\}
$$

is a dense set in $\boldsymbol{V}_{y} \times H_{y}^{1}(D)$. Finally, we have to ensure that the integral

$$
\int_{0}^{T} \int_{D}\left[\left(\left(\boldsymbol{u} \cdot \nabla_{h}\right) \mathbf{u}\right) \cdot \boldsymbol{\psi}-\left(\left(\boldsymbol{u} \cdot \nabla_{h}\right) \boldsymbol{\psi}\right) \cdot \boldsymbol{u}\right] h^{2} y d(x, y) d t
$$

over the nonlinear items remains bounded during the approximation of $\psi$ by smooth functions. In the proof of the second a priori estimate, we obtained boundedness of similar integrals (5.14) by the use of the weight function $y^{\lambda+1}$ instead of $y$, which ensures existence and boundedness of the integrals. In the case of relation (6.2), we forego some additional 
weight; however, we assume some more regularity $\psi \in L^{5}\left(0, T ; H_{y}^{1,5}(D)^{2}\right)$ which especially controls the possible singularity of $\boldsymbol{\psi}$ at $y=0$. From (2.29) we obtain $\boldsymbol{u} \in L^{p}\left(0, T ; L_{y}^{p}(D)^{2}\right)$ for $p=10 / 3$. Hence Hölder's inequality together with $\boldsymbol{u} \in L^{2}\left(0, T ; H_{y}^{1}(D)^{2}\right)$ implies boundedness of (6.7) if we approximate $\psi$ in $L^{r}\left(0, T ; H_{y}^{1, r}(D)^{2}\right)$ for $r=2 p /(p-2)=5$ by smooth functions.

Therefore we find a sequence of smooth functions which approximates the test function $(\psi, \omega, \xi)$ given in Definition 6.1 and transfers the relation (6.2) for smooth test functions into relation (6.2) with $(\psi, \omega, \xi)$ belonging to the given function spaces. Then it is obvious that

$$
\left(v_{x}(x, r, t), v_{r}(x, r, t)\right)=\boldsymbol{u}(x, r / h, t), \quad p(x, r, t)=q(x, r / h, t)
$$

is a solution of problem (1.1)-(1.14) in the sense of Definition 6.1 if $h=h(\eta)$ is defined by (1.4) with $\eta$ given by (5.32). The estimates (1.20) follow for sufficiently small $T>0$ ( $c f$. (4.7)) from Theorems 4.1 and 4.4 .

\section{Linearisation}

Assume now that

$$
\boldsymbol{u}^{\bullet} \in L^{\infty}\left(0, T ; L_{y}^{2}(D)^{2}\right) \cap L^{2}\left(0, T ; \boldsymbol{V}_{y}\right) \quad \text { is given. }
$$

We investigate next the system

$$
\begin{aligned}
& \rho \frac{\partial v_{x}}{\partial t}+\rho v_{x}^{\bullet} \frac{\partial v_{x}}{\partial x}+\rho v_{r}^{\bullet} \frac{\partial v_{x}}{\partial r}+\frac{\rho}{2} v_{x}\left(\frac{\partial v_{x}^{\bullet}}{\partial x}+\frac{\partial v_{r}^{\bullet}}{\partial r}+\frac{1}{r} v_{r}^{\bullet}\right) \\
& =\mu\left\{2 \frac{\partial^{2} v_{x}}{\partial x^{2}}+\frac{1}{r} \frac{\partial}{\partial r}\left(r\left(\frac{\partial v_{x}}{\partial r}+\frac{\partial v_{r}}{\partial x}\right)\right)\right\}-\frac{\partial p}{\partial x}, \\
& \rho \frac{\partial v_{r}}{\partial t}+\rho v_{x}^{\bullet} \frac{\partial v_{r}}{\partial x}+\rho v_{r}^{\bullet} \frac{\partial v_{r}}{\partial r}+\frac{\rho}{2} v_{r}\left(\frac{\partial v_{x}^{\bullet}}{\partial x}+\frac{\partial v_{r}^{\bullet}}{\partial r}+\frac{1}{r} v_{r}^{\bullet}\right) \\
& =\mu\left\{\frac{2}{r} \frac{\partial}{\partial r}\left(r \frac{\partial v_{r}}{\partial r}\right)+\frac{\partial}{\partial x}\left(\frac{\partial v_{r}}{\partial x}+\frac{\partial v_{x}}{\partial r}\right)-\frac{2}{r^{2}} v_{r}\right\}-\frac{\partial p}{\partial r}, \\
& \varepsilon\left(\frac{\partial p}{\partial t}-\frac{\partial^{2} p}{\partial x^{2}}-\frac{1}{r} \frac{\partial}{\partial r}\left(r \frac{\partial p}{\partial r}\right)\right)+\frac{\partial v_{x}}{\partial x}+\frac{\partial v_{r}}{\partial r}+\frac{1}{r} v_{r}=0
\end{aligned}
$$

in $\Omega(h),(1.5)$,

$$
\begin{gathered}
-E\left[\frac{\partial^{2} \eta}{\partial t^{2}}-g \frac{\partial^{6} \eta}{\partial x^{6}}+e \frac{\partial^{5} \eta}{\partial t^{3} \partial x^{2}}+d \frac{\partial^{5} \eta}{\partial t \partial x^{4}}+f \frac{\partial^{4} \eta}{\partial x^{4}}-c \frac{\partial^{3} \eta}{\partial t \partial x^{2}}-a \frac{\partial^{2} \eta}{\partial x^{2}}+b \eta\right] \\
=h\left[2 \mu \frac{\partial v_{r}}{\partial r}+\mu\left(\frac{\partial v_{r}}{\partial x}+\frac{\partial v_{x}}{\partial r}\right)\left(-\frac{\partial h}{\partial x}\right)-p+p_{w}-\frac{\rho}{2} v_{r}\left(v_{r}^{\bullet}-\frac{\partial h}{\partial t}\right)\right]
\end{gathered}
$$

for $0<x<L, 0<t<T$,

$$
\eta(0, t)=\frac{\partial \eta}{\partial x}(0, t)=\frac{\partial^{2} \eta}{\partial x^{2}}(0, t)=\eta(L, t)=\frac{\partial \eta}{\partial x}(L, t)=\frac{\partial^{2} \eta}{\partial x^{2}}(L, t)=0
$$

for any $0<t<T$, (1.8)-(1.10),

$$
\left(2 \mu \frac{\partial v_{x}}{\partial x}-p-p_{\text {out }}-\frac{\rho}{2} v_{x}^{\bullet} v_{x}\right)\left(L^{+}, r, t\right)=0, \quad v_{r}\left(L^{+}, r, t\right)=0
$$


for any $0<r<R^{+}, 0<t<T$,

$$
\left(2 \mu \frac{\partial v_{x}}{\partial x}-p+p_{\text {in }}-\frac{\rho}{2} v_{x}^{\cdot} v_{x}\right)\left(-L^{-}, r, t\right)=0, \quad v_{r}\left(-L^{-}, r, t\right)=0
$$

for any $0<r<R^{-}, 0<t<T$ and (1.13)-(1.14), where

$$
\left(v_{x}^{\bullet}(x, r, t), v_{r}^{\bullet}(x, r, t)\right)=\boldsymbol{u}^{\bullet}\left(x, \frac{r}{h(x, t)}, t\right) .
$$

Our ultimate goal was of course to consider Navier-Stokes equations. Due to estimate (5.4) of Theorem 5.1 that unfortunately depends on $\varepsilon$, we do not have compactness with respect to $t$. For linearised Navier-Stokes equations, however, weak convergence of velocity suffices.

We can now repeatedly apply the foregoing considerations to ensure an existence result for the above problem. Note, however, that for given $0<\delta \ll 1$, we replace $\left(v_{x}^{\bullet}, v_{r}^{\bullet}\right)$ givenby (7.7) by

$$
\left(\left(v_{x}^{\bullet}\right)_{\delta}(x, r, t),\left(v_{r}^{\bullet}\right)_{\delta}(x, r, t)\right)=\boldsymbol{u}^{\bullet}\left(x, \frac{r-\delta}{h(x, t)-\delta}, t\right) .
$$

Thus, there exists a solution $\left(\boldsymbol{u}^{\varepsilon}, q^{\varepsilon}, \eta^{\varepsilon}\right)$ of (7.2)-(7.6) in the sense of the validity of the following integral identity:

$$
\begin{aligned}
-\rho & \int_{0}^{T} \int_{D} \boldsymbol{u}^{\varepsilon} \frac{\partial \boldsymbol{\psi}}{\partial t}\left(h^{\varepsilon}\right)^{2} y d(x, y) d t-\varepsilon \int_{0}^{T} \int_{D} q^{\varepsilon} \frac{\partial \omega}{\partial t}\left(h^{\varepsilon}\right)^{2} y d(x, y) d t \\
& -\rho \int_{0}^{T} \int_{D}\left\{\boldsymbol{u}^{\varepsilon} \cdot \boldsymbol{\psi}+\left[\frac{\partial \boldsymbol{u}^{\varepsilon}}{\partial y} \cdot \boldsymbol{\psi}-\boldsymbol{u}^{\varepsilon} \cdot \frac{\partial \boldsymbol{\psi}}{\partial y}\right] \frac{y}{2}\right\} h^{\varepsilon} \frac{\partial h^{\varepsilon}}{\partial t} y d(x, y) d t \\
& -\frac{\varepsilon}{2} \int_{0}^{T} \int_{D}\left\{q^{\varepsilon} \omega 2 h^{\varepsilon} \frac{\partial h^{\varepsilon}}{\partial t} y+\left[\frac{\partial q^{\varepsilon}}{\partial y} \omega-q^{\varepsilon} \frac{\partial \omega}{\partial y}\right] h^{\varepsilon} \frac{\partial h^{\varepsilon}}{\partial t} y^{2}\right\} d(x, y) d t \\
& +\int_{0}^{T} \int_{D}\left\{\frac{\rho}{2}\left[\left(\left(\boldsymbol{u}^{\bullet} \cdot \nabla_{h^{\varepsilon}}\right) \boldsymbol{u}^{\varepsilon}\right) \cdot \boldsymbol{\psi}-\left(\left(\boldsymbol{u}^{\bullet} \cdot \nabla_{h^{\varepsilon}}\right) \boldsymbol{\psi}\right) \cdot \mathbf{u}^{\varepsilon}\right]\right. \\
& \left.+\mu\left(\left[\nabla_{h^{\varepsilon}} \boldsymbol{u}^{\varepsilon}+\left(\nabla_{h^{\varepsilon}} \boldsymbol{u}^{\varepsilon}\right)^{T}\right]: \nabla_{h^{\varepsilon}} \boldsymbol{\psi}+\frac{2}{y^{2}} u_{2}^{\varepsilon} \psi_{2}\right)\right\}\left(h^{\varepsilon}\right)^{2} y d(x, y) d t \\
& +\int_{0}^{T} \int_{D}\left\{\varepsilon \nabla_{h^{\varepsilon}} q^{\varepsilon} \cdot \nabla_{h^{\varepsilon}} \omega+\omega \operatorname{div} h_{h^{\varepsilon}} \boldsymbol{u}^{\varepsilon}-q^{\varepsilon} \operatorname{div}_{h^{\varepsilon}} \boldsymbol{\psi}\right\}\left(h^{\varepsilon}\right)^{2} y d(x, y) d t \\
& +E \int_{0}^{T} \int_{0}^{L}\left\{-\eta_{t}^{\varepsilon} \xi_{t}+g \eta_{x x x}^{\varepsilon} \xi_{x x x}+d \eta_{t x x}^{\varepsilon} \xi_{x x}+e \eta_{t x t}^{\varepsilon} \xi_{x t}\right. \\
& \left.+f \eta_{x x}^{\varepsilon} \xi_{x x}+c \eta_{x t}^{\varepsilon} \xi_{x}+a \eta_{x}^{\varepsilon} \xi_{x}+b \eta^{\varepsilon} \xi\right\} d x d t \\
& +\int_{0}^{T} \int_{0}^{L} q_{w} \psi_{2}\left(h^{\varepsilon}\right)^{2} d x d t \\
= & \int_{0}^{T} \int_{0}^{1}\left(\left(q_{\text {in }} \psi_{1}\left(h^{\varepsilon}\right)^{2}\right)\left(-L^{-}, y, t\right)+\left(q_{\text {out }} \psi_{1}\left(h^{\varepsilon}\right)^{2}\right)\left(L^{+}, y, t\right)\right) y d y d t
\end{aligned}
$$

for every $(\boldsymbol{\psi}, \omega, \xi) \in L^{2}\left(0, T ; V_{y}\right), \boldsymbol{\psi} \in L^{5}\left(0, T ; H_{y}^{1,5}(D)^{2}\right) \cap H^{1,1}\left(0, T ; L_{y}^{2}(D)^{2}\right), \omega \in H^{1,1}(0, T$; $\left.L_{y}^{2}(D)\right), \xi \in H^{1,1}\left(0, T ; H_{0}^{1}(0, L)\right)$ with $(\psi, \omega, \xi)(\cdot, T)=\mathbf{0}$ and $\psi_{2}=\xi$ a.e. on $(0, T) \times\left(S_{w} \backslash \Gamma\right)$. 
Note that $\left(\boldsymbol{u}^{\varepsilon}, q^{\varepsilon}, \eta^{\varepsilon}\right)$ inherits from (3.1), by the presence of (1.15), the following a priori estimate:

$$
\begin{aligned}
& \int_{D}\left(\rho\left|\boldsymbol{u}^{\varepsilon}(t)\right|^{2}+\varepsilon\left|q^{\varepsilon}(t)\right|^{2}\right)\left(h^{\varepsilon}(t)\right)^{2} y d(x, y) \\
& \quad+\frac{\alpha^{2}}{4\left(K^{2}+2\right)} \int_{0}^{t} \int_{D}\left(\mu\left|\nabla \boldsymbol{u}^{\varepsilon}\right|^{2}+\varepsilon\left|\nabla q^{\varepsilon}\right|^{2}\right)\left(h^{\varepsilon}(s)\right)^{2} y d(x, y) d s \\
& \quad+2 \mu \int_{0}^{t} \int_{D} \frac{1}{y}\left|u_{2}^{\varepsilon}\right|^{2} d(x, y) d s \\
& \quad+E \int_{0}^{L}\left(\left|\eta_{t}^{\varepsilon}\right|^{2}+g\left|\eta_{x x x}^{\varepsilon}\right|^{2}+a\left|\eta_{x}^{\varepsilon}\right|^{2}+b\left|\eta^{\varepsilon}\right|^{2}+f\left|\eta_{x x}^{\varepsilon}\right|^{2}\right)(x, t) d x \\
& \quad+2 E \int_{0}^{t} \int_{0}^{L}\left(d\left|\eta_{t x x}\right|^{2}+e\left|\eta_{t x t}^{\varepsilon}\right|^{2}+c\left|\eta_{t x}^{\varepsilon}\right|^{2}\right) d x d s \\
& \leq C .
\end{aligned}
$$

The constant $C$ does not depend on $\varepsilon$. Therefore, there exist $(\boldsymbol{u}, \eta)$ and a subsequence of $\left(\boldsymbol{u}^{\varepsilon}, \eta^{\varepsilon}\right)$ such that

$$
\begin{aligned}
& y \mathbf{u}^{\varepsilon} \rightarrow y \boldsymbol{u} \quad \text { weakly in } L^{2}\left(0, T ; L^{2}(D)^{2}\right), \\
& y^{-1 / 2} u_{2}^{\varepsilon} \rightarrow y^{-1 / 2} u_{2} \quad \text { weakly in } L^{2}\left(0, T ; L^{2}(D)\right), \\
& y \nabla \boldsymbol{u}^{\varepsilon} \rightarrow y \nabla \boldsymbol{u} \quad \text { weakly in } L^{2}\left(0, T ; L^{2}(D)^{2}\right), \\
& y \nabla_{h^{\varepsilon}} \boldsymbol{u}^{\varepsilon} \rightarrow y \nabla_{h} \boldsymbol{u} \quad \text { weakly in } L^{2}\left(0, T ; L^{2}(D)^{2}\right), \\
& \eta_{x x x}^{\varepsilon} \rightarrow \eta_{x x x} \quad \text { weakly in } L^{2}\left(0, T ; L^{2}(0, L)\right), \\
& \eta_{t x x}^{\varepsilon} \rightarrow \eta_{t x x} \quad \text { weakly in } L^{2}\left(0, T ; L^{2}(0, L)\right), \\
& \eta_{t x t}^{\varepsilon} \rightarrow \eta_{t x t} \quad \text { weakly in } L^{2}\left(0, T ; L^{2}(0, L)\right), \\
& \eta_{x x}^{\varepsilon} \rightarrow \eta_{x x} \quad \text { strongly in } L^{2}\left(0, T ; L^{2}(0, L)\right), \\
& \eta_{t}^{\varepsilon} \rightarrow \eta_{t} \quad \text { strongly in } L^{2}\left(0, T ; L^{2}(0, L)\right), \\
& \eta_{x t}^{\varepsilon} \rightarrow \eta_{x t} \quad \text { strongly in } L^{2}\left(0, T ; L^{2}(0, L)\right), \\
& \eta^{\varepsilon} \rightarrow \eta \quad \text { in } C^{1}([0, T] \times[0, L]), \\
& h^{\varepsilon}=h\left(\eta^{\varepsilon}\right) \rightarrow h \stackrel{\text { def }}{=} h(\eta) \quad \text { in } C^{1}([0, T] \times[0, L]) \text { as } \varepsilon \rightarrow 0 .
\end{aligned}
$$

Owing to (7.9) for $q^{\varepsilon}$, we deduce

$$
\operatorname{div}_{h} u=0 \quad \text { a.e. on } D \times(0, T) .
$$

To see (7.11), let us insert $(\boldsymbol{\psi}, \omega, \xi)=(0, \omega, 0)$ into $(7.8)$ to get

$$
\begin{aligned}
\int_{0}^{T} \int_{D} \omega\left(\operatorname{div}_{h^{\varepsilon}} \boldsymbol{u}^{\varepsilon}\right)\left(h^{\varepsilon}\right)^{2} y d(x, y) d t= & \sqrt{\varepsilon} \int_{0}^{T} \int_{D}\left\{\sqrt{\varepsilon} q^{\varepsilon} \omega \frac{\partial\left(h^{\varepsilon} \omega\right)}{\partial t}-\sqrt{\varepsilon} \nabla_{h^{\varepsilon}} q^{\varepsilon} \cdot \nabla_{h^{\varepsilon}} \omega\right. \\
& \left.+\sqrt{\varepsilon}\left[\frac{\partial q^{\varepsilon}}{\partial y} \omega-q^{\varepsilon} \frac{\partial \omega}{\partial y}\right] \frac{y}{2} \frac{\partial h^{\varepsilon}}{\partial t}\right\} h^{\varepsilon} y d(x, y) d t \rightarrow 0
\end{aligned}
$$

As $\omega$ was arbitrary, (7.11) holds. 
To get the limiting behaviour of the left-hand side of (7.8) under control, we have to work with $h^{\varepsilon}$-divergence-free test functions, i.e. with functions $\boldsymbol{\psi}^{\varepsilon}$ satisfying (1.18). We insert this deduction together with $\omega \equiv 0$ into (7.8) and simplify:

$$
\begin{aligned}
-\rho & \int_{0}^{T} \int_{D} \boldsymbol{u}^{\varepsilon} \frac{\partial \boldsymbol{\psi}^{\varepsilon}}{\partial t}\left(h^{\varepsilon}\right)^{2} y d(x, y) d t \\
& -\rho \int_{0}^{T} \int_{D}\left\{\boldsymbol{u}^{\varepsilon} \cdot \boldsymbol{\psi}^{\varepsilon}+\left[\frac{\partial \boldsymbol{u}^{\varepsilon}}{\partial y} \cdot \boldsymbol{\psi}^{\varepsilon}-\boldsymbol{u}^{\varepsilon} \cdot \frac{\partial \boldsymbol{\psi}^{\varepsilon}}{\partial y}\right] \frac{y}{2}\right\} h^{\varepsilon} \frac{\partial h^{\varepsilon}}{\partial t} y d(x, y) d t \\
& +\int_{0}^{T} \int_{D}\left\{\frac{\rho}{2}\left[\left(\left(\boldsymbol{u}^{\bullet} \cdot \nabla_{h^{\varepsilon}}\right) \boldsymbol{u}^{\varepsilon}\right) \cdot \boldsymbol{\psi}^{\varepsilon}-\left(\left(\boldsymbol{u}^{\bullet} \cdot \nabla_{h^{\varepsilon}}\right) \boldsymbol{\psi}^{\varepsilon}\right) \cdot \boldsymbol{u}^{\varepsilon}\right]\right. \\
& \left.+\mu\left(\left[\nabla_{h^{\varepsilon}} \boldsymbol{u}^{\varepsilon}+\left(\nabla_{h^{\varepsilon}} \boldsymbol{u}^{\varepsilon}\right)^{T}\right]: \nabla_{h^{\varepsilon}} \boldsymbol{\psi}^{\varepsilon}+\frac{2}{y^{2}} u_{2}^{\varepsilon} \psi_{2}^{\varepsilon}\right)\right\}\left(h^{\varepsilon}\right)^{2} y d(x, y) d t \\
& +E \int_{0}^{T} \int_{0}^{L}\left\{-\eta_{t}^{\varepsilon} \xi_{t}^{\varepsilon}+g \eta_{x x x}^{\varepsilon} \xi_{x x x}^{\varepsilon}+d \eta_{t x x}^{\varepsilon} \xi_{x x}^{\varepsilon}+e \eta_{t x t}^{\varepsilon} \xi_{x t}^{\varepsilon}\right. \\
& \left.+f \eta_{x x}^{\varepsilon} \xi_{x x}^{\varepsilon}+c \eta_{x t}^{\varepsilon} \xi_{x}^{\varepsilon}+a \eta_{x}^{\varepsilon} \xi_{x}^{\varepsilon}+b \eta^{\varepsilon} \xi^{\varepsilon}\right\} d x d t \\
& +\int_{0}^{T} \int_{0}^{L} q_{w} \psi_{2}^{\varepsilon}\left(h^{\varepsilon}\right)^{2} d x d t \\
= & \int_{0}^{T} \int_{0}^{1}\left(\left(q_{\text {in }} \psi_{1}^{\varepsilon}\left(h^{\varepsilon}\right)^{2}\right)\left(-L^{-}, y, t\right)+\left(q_{\text {out }} \psi_{1}^{\varepsilon}\left(h^{\varepsilon}\right)^{2}\right)\left(L^{+}, y, t\right)\right) y d y d t .
\end{aligned}
$$

According to (7.10), we have to suppose that

$$
\begin{aligned}
& \boldsymbol{\psi}^{\varepsilon} \rightarrow \boldsymbol{\psi} \quad \text { strongly in } H^{1}(D \times(0, T)), \\
& \nabla_{h^{\varepsilon}} \boldsymbol{\psi}^{\varepsilon} \rightarrow \nabla_{h} \boldsymbol{\psi} \quad \text { strongly in } L^{2}\left(0, T ; L^{2}(D)^{2}\right)^{2}, \\
& \xi_{x x x}^{\varepsilon} \rightarrow \xi_{x x x} \quad \text { strongly in } L^{2}\left(0, T ; L^{2}(0, L)\right), \\
& \xi^{\varepsilon} \rightarrow \xi \quad \text { strongly in } H^{2}((0, L) \times(0, T)),
\end{aligned}
$$

where

$$
\xi^{\varepsilon}(x, t)=\psi_{2}^{\varepsilon}(x, 1, t)
$$

Our goal now is to verify that $\boldsymbol{\psi}^{\varepsilon}$ given by (1.19) fulfil all the requirements we need. This is true if we make this hypothesis: Suppose $\phi \in C^{3}(\bar{D} \times[0, T])$,

$$
\begin{aligned}
& |\phi| \leq C y^{\omega}, \quad \omega>5 / 3 \quad \text { for }(x, y, t) \in \bar{D} \times[0, T], \\
& \frac{\partial \phi}{\partial x}=\frac{\partial \phi}{\partial y}=\phi=0 \quad \text { for } t=T,(x, y) \in \bar{D} \text {, } \\
& \frac{\partial \phi}{\partial y}=0 \quad \text { for } y=1,(x, t) \in\left[-L^{-}, L^{+}\right] \times[0, T], \\
& \frac{\partial \phi}{\partial x}=0 \quad \text { for } x=-L^{-}, y \in[0,1], t \in[0, T], \\
& \text { for } x=L^{+}, y \in[0,1], t \in[0, T] \text {, } \\
& \int_{0}^{1} \phi(x, z, t) d z=0 \quad \text { for } x \in\left[-L^{-}, L^{+}\right], t \in[0, T], \\
& \phi=\frac{\partial \phi}{\partial x}=\frac{\partial^{2} \phi}{\partial x^{2}}=0 \quad \text { for } y=1, x \in\left[-L^{-}, 0\right] \cup\left[0, L^{+}\right], t \in[0, T]
\end{aligned}
$$


for some $C>0$. It is now straightforward to check that $\psi^{\varepsilon} \in L^{2}\left(0, T ; \boldsymbol{V}_{y}\right) \cap L^{5}(0, T$; $\left.H_{y}^{1,5}(D)^{2}\right) \cap H^{1,1}\left(0, T ; L_{y}^{2}(D)^{2}\right)$ satisfies (1.18) and

$$
\xi^{\varepsilon}(x, t) \stackrel{\text { def }}{=} \psi_{2}^{\varepsilon}(x, 1, t)=\phi(x, 1, t) \in C^{3}([0, L] \times[0, T])
$$

with $(\boldsymbol{\psi}, \xi)(\cdot, T)=\mathbf{0}$.

We have just proved that the set of appropriate test functions satisfying constraint (1.18) is not empty, and due to (7.10), (7.13), we can let $\varepsilon \rightarrow 0$ in (7.12) to arrive at

$$
\begin{aligned}
-\rho & \int_{0}^{T} \int_{D} \boldsymbol{u} \frac{\partial \boldsymbol{\psi}}{\partial t} h^{2} y d(x, y) d t \\
& -\rho \int_{0}^{T} \int_{D}\left\{\boldsymbol{u} \cdot \boldsymbol{\psi}+\left[\frac{\partial \boldsymbol{u}}{\partial y} \cdot \boldsymbol{\psi}-\boldsymbol{u} \cdot \frac{\partial \boldsymbol{\psi}}{\partial y}\right] \frac{y}{2}\right\} h \frac{\partial h}{\partial t} y d(x, y) d t \\
& +\int_{0}^{T} \int_{D}\left\{\frac{\rho}{2}\left[\left(\left(\boldsymbol{u}^{\bullet} \cdot \nabla_{h}\right) \boldsymbol{u}\right) \cdot \boldsymbol{\psi}-\left(\left(\boldsymbol{u}^{\bullet} \cdot \nabla_{h}\right) \boldsymbol{\psi}\right) \cdot \boldsymbol{u}\right]\right. \\
& \left.+\mu\left(\left[\nabla_{h} \boldsymbol{u}+\left(\nabla_{h} \boldsymbol{u}\right)^{T}\right]: \nabla_{h} \boldsymbol{\psi}+\frac{2}{y^{2}} u_{2} \psi_{2}\right)\right\} h^{2} y d(x, y) d t \\
& +E \int_{0}^{T} \int_{0}^{L}\left\{-\eta_{t} \xi_{t}+g \eta_{x x x} \xi_{x x x}+d \eta_{t x x} \xi_{x x}+e \eta_{t x t} \xi_{x t}\right. \\
& \left.+f \eta_{x x} \xi_{x x}+c \eta_{x t} \xi_{x}+a \eta_{x} \xi_{x}+b \eta \xi\right\} d x d t \\
& +\int_{0}^{T} \int_{0}^{L} q_{w} \psi_{2} h^{2} d x d t \\
= & \int_{0}^{T} \int_{0}^{1}\left(\left(q_{\text {in }} \psi_{1} h^{2}\right)\left(-L^{-}, y, t\right)+\left(q_{\text {out }} \psi_{1} h^{2}\right)\left(L^{+}, y, t\right)\right) y d y d t
\end{aligned}
$$

that holds for any $\psi \in L^{2}\left(0, T ; \boldsymbol{V}_{y}\right) \cap L^{5}\left(0, T ; H_{y}^{1,5}(D)^{2}\right) \cap H^{1}\left(0, T ; L_{y}^{2}(D)^{2}\right)$,

$$
\operatorname{div}_{h}(\boldsymbol{\psi})=0, \quad \xi(x, t)=\psi_{2}(x, 1, t) \in L^{2}\left(0, T ; H_{0}^{3}(0, L)\right) \cap H^{1}\left(0, T ; H_{0}^{1}(0, L)\right)
$$

with $(\boldsymbol{\psi}, \xi)(\cdot, T)=\mathbf{0}$.

Making use of (7.15), a corresponding existence result for (7.2)-(7.6) with $\varepsilon=0$ can be formulated.

Theorem 7.1 Let $\boldsymbol{u}^{\bullet}$ be given by (7.1). Suppose that the data satisfy (2.20) and assume $0<\alpha \ll 1$ such small that $4 \alpha<R^{+}+R^{-}<\alpha^{-1}$, and let $K=R^{+}-2 \alpha+\left\|\chi^{\prime}\right\|_{\infty}$.

Then there is $T>0$ and a weak solution $\left(v_{x}, v_{r}, \eta\right)$ of problem (7.2)-(7.7) with $\varepsilon=0$ on $[0, T]$ such that

$$
\eta \in W^{1, \infty}((0, L) \times(0, T)), \quad \eta_{t}(x, t)=v_{r}(x, h(x, t), t),
$$

where $h$ is defined by (1.4) in terms of the component $\eta$ and such that the couple $(\boldsymbol{u}, \eta)$ given by (1.16) has the following properties:

(1) $\boldsymbol{u} \in L^{\infty}\left(0, T ; L_{y}^{2}(D)^{2}\right) \cap L^{2}\left(0, T ; \boldsymbol{V}_{y}\right)$,

(2) $\operatorname{div}_{h} \boldsymbol{u}=0$ a.e. on $D \times(0, T)$,

(3) $\eta \in H_{0-}^{1}\left(0, T ; H_{0}^{2}(0, L)\right) \cap H_{0-}^{2}\left(0, T ; H_{0}^{1}(0, L)\right) \cap L^{2}\left(0, T ; H_{0}^{3}(0, L)\right)$,

(4) and $(\boldsymbol{u}, \eta)$ satisfies the identity (7.15). 


\section{Appendix}

In this appendix we want to present some ideas of the proof of Theorem 4.3. Note that the regularising parameters $\varepsilon, \kappa$ and $\delta$ are fixed.

We start with two weak solutions $\left(\boldsymbol{u}^{1}, q^{1}, z^{1}\right)$ and $\left(\boldsymbol{u}^{2}, q^{2}, z^{2}\right)$ of the initial boundary value problem (2.9)-(2.17) corresponding to the given data $h^{1}=h\left(\bar{\eta}^{1}\right), q_{\text {in }}^{1}, q_{w}^{1}, q_{\text {out }}^{1}$ and $h^{2}=h\left(\bar{\eta}^{2}\right)$, $q_{\mathrm{in}}^{2}, q_{w}^{2}, q_{\mathrm{out}}^{2}$, respectively, and form the difference of the appropriate relations (2.21). As a test function, we insert

$$
(\psi(\cdot, \cdot, s), \omega(\cdot, \cdot, s), \xi(\cdot, s))= \begin{cases}\left(\mathbf{u}^{1} \ell^{1}-\mathbf{u}^{2} \ell^{2}, q^{1} \ell^{1}-q^{2} \ell^{2}, z^{1}-z^{2}\right) & \text { for } \tau \leq s \leq t, \\ 0 & \text { otherwise }\end{cases}
$$

where $\tau<t$ are fixed in $[0, T]$ and $\ell^{i}, i=1,2$, are defined by (2.10) with $h=h^{i}$. Then some items of the resulting relation are

$$
\begin{aligned}
& \int_{\tau}^{t}\left\{\rho\left\langle\frac{\partial\left(\boldsymbol{u}^{1} \ell^{1}-\boldsymbol{u}^{2} \ell^{2}\right)}{\partial t}, \boldsymbol{u}^{1} \ell^{1}-\boldsymbol{u}^{2} \ell^{2}\right\rangle+\varepsilon\left\langle\frac{\partial\left(q^{1} \ell^{1}-q^{2} \ell^{2}\right)}{\partial t}, q^{1} \ell^{1}-q^{2} \ell^{2}\right\rangle\right\} d s \\
& -\frac{\rho}{2} \int_{\tau}^{t} \int_{D}\left\{\left(\boldsymbol{u}^{1} \cdot\left(\mathbf{u}^{1} \ell^{1}-\boldsymbol{u}^{2} \ell^{2}\right)\right) \frac{\partial \ell^{1}}{\partial t}-\left(\boldsymbol{u}^{2} \cdot\left(\boldsymbol{u}^{1} \ell^{1}-\boldsymbol{u}^{2} \ell^{2}\right)\right) \frac{\partial \ell^{2}}{\partial t}\right\} d(x, y) d s \\
& -\frac{\rho}{2} \int_{\tau}^{t} \int_{D}\left\{\left[\frac{\partial \mathbf{u}^{1}}{\partial y} \cdot\left(\mathbf{u}^{1} \ell^{1}-\mathbf{u}^{2} \ell^{2}\right)-\mathbf{u}^{1} \cdot \frac{\partial}{\partial y}\left(\mathbf{u}^{1} \ell^{1}-\mathbf{u}^{2} \ell^{2}\right)\right]\left[\left(h^{1}-\delta\right) y+\delta\right] \frac{\partial h^{1}}{\partial t} y\right. \\
& \left.-\left[\frac{\partial \boldsymbol{u}^{2}}{\partial y} \cdot\left(\boldsymbol{u}^{1} \ell^{1}-\boldsymbol{u}^{2} \ell^{2}\right)-\boldsymbol{u}^{2} \cdot \frac{\partial}{\partial y}\left(\boldsymbol{u}^{1} \ell^{1}-\boldsymbol{u}^{2} \ell^{2}\right)\right]\left[\left(h^{2}-\delta\right) y+\delta\right] \frac{\partial h^{2}}{\partial t} y\right\} d(x, y) d s \\
& \text { : } \\
& +\int_{\tau}^{t} \int_{D} \frac{\rho}{2}\left\{\left[\left(\left(\boldsymbol{u}^{1} \cdot \nabla_{h^{1}}\right) \boldsymbol{u}^{1}\right) \cdot\left(\boldsymbol{u}^{1} \ell^{1}-\boldsymbol{u}^{2} \ell^{2}\right)-\left(\left(\boldsymbol{u}^{1} \cdot \nabla_{h^{1}}\right)\left(\boldsymbol{u}^{1} \ell^{1}-\boldsymbol{u}^{2} \ell^{2}\right)\right) \cdot \mathbf{u}^{1}\right] \ell^{1}\right. \\
& \left.-\left[\left(\left(\boldsymbol{u}^{2} \cdot \nabla_{h^{2}}\right) \mathbf{u}^{2}\right) \cdot\left(\boldsymbol{u}^{1} \ell^{1}-\boldsymbol{u}^{2} \ell^{2}\right)-\left(\left(\boldsymbol{u}^{2} \cdot \nabla_{h^{2}}\right)\left(\boldsymbol{u}^{1} \ell^{1}-\boldsymbol{u}^{2} \ell^{2}\right)\right) \cdot \mathbf{u}^{2}\right] \ell^{2}\right\} d(x, y) d s \\
& \vdots \\
& +\kappa \int_{\tau}^{t} \int_{0}^{L}\left(\left(u_{2}^{1}-z^{1}\right)-\left(u_{2}^{2}-z^{2}\right)\right)\left(\left(u_{2}^{1} \ell^{1}-u_{2}^{2} \ell^{2}\right)-\left(z^{1}-z^{2}\right)\right) d x d s \\
& =\int_{\tau}^{t} \int_{0}^{1}\left\{\left(q_{\text {in }}^{1} \ell^{1}-q_{\text {in }}^{2} \ell^{2}\right)\left(\boldsymbol{u}^{1} \ell^{1}-\boldsymbol{u}^{2} \ell^{2}\right)\left(-L^{-}, y, t\right)\right. \\
& \left.+\left(q_{\text {out }}^{1} \ell^{1}-q_{\text {out }}^{2} \ell^{2}\right)\left(\boldsymbol{u}^{1} \ell^{1}-\mathbf{u}^{2} \ell^{2}\right)\left(L^{+}, y, t\right)\right\} d y d t .
\end{aligned}
$$

Now, we denote

$$
\boldsymbol{U}=\boldsymbol{u}^{1} \ell^{1}-\boldsymbol{u}^{2} \ell^{2}, \quad Q=q^{1} \ell^{1}-q^{2} \ell^{2}, \quad Z=Z^{1}-Z^{2}, \quad z=z^{1}-z^{2} .
$$

Then the aim of the proof is to derive an integral inequality of the form

$$
\begin{aligned}
& \rho \int_{D}|\boldsymbol{U}(x, y, t)|^{2} d(x, y)+v_{1} \int_{\tau}^{t} \int_{D}|\nabla \boldsymbol{U}(x, y, s)|^{2} d(x, y) d s \\
& \quad+\varepsilon \int_{D}|Q(x, y, t)|^{2} d(x, y)+v_{2} \varepsilon \int_{\tau}^{t} \int_{D}|\nabla Q(x, y, s)|^{2} d(x, y) d s
\end{aligned}
$$




$$
\begin{aligned}
& +E \int_{0}^{L}\left\{|z(x, t)|^{2}+f\left|Z_{x x}(x, t)\right|^{2}+a\left|Z_{x}(x, t)\right|^{2}+b|Z(x, t)|^{2}\right\} d x \\
& +E \int_{\tau}^{t} \int_{0}^{L}\left\{d\left|z_{x x}(x, s)\right|^{2}+e\left|z_{x t}(x, s)\right|^{2}+c\left|z_{x}(x, s)\right|^{2}\right\} d x d s \\
& +\alpha^{2} \kappa \int_{\tau}^{t} \int_{0}^{L}\left|U_{2}(x, s)\right|^{2} d x d s \\
& \leq \sigma \int_{\tau}^{t} \int_{D}\left(|\nabla \boldsymbol{U}|^{2}+\varepsilon|\nabla Q|^{2}\right) d(x, y) d s+\sigma \int_{\tau}^{t} \int_{0}^{L}\left|U_{2}(x, s)\right|^{2} d x d s \\
& +\int_{\tau}^{t} \beta(s) \int_{D}|\boldsymbol{U}(x, y, s)|^{2} d(x, y) d s \\
& +c_{\sigma} \varepsilon \int_{\tau}^{t} \int_{D}|Q(x, y, s)|^{2} d(x, y) d s+c_{\sigma} \int_{\tau}^{t} \int_{0}^{L}|z(x, s)|^{2} d x d s \\
& +c_{\sigma} \sum_{i=1}^{2}\left(\varepsilon\left\|q^{i}\right\|_{L^{2}\left(\tau, t ; L^{2}(D)\right)}^{2}+\left\|\boldsymbol{u}^{i}\right\|_{L^{2}\left(\tau, t ; H^{1}(D)\right)}^{2}\right)\left\|h^{1}-h^{2}\right\|_{W^{1, \infty}\left(\mathcal{S}_{\tau, t}\right)}^{2} \\
& +c_{\sigma} \int_{\tau}^{t}\left(\left\|q_{\text {out }}^{1}-q_{\text {out }}^{2}\right\|_{L^{2}\left(S_{\text {out }}\right)}^{2}+\left\|q_{\text {in }}^{1}-q_{\text {in }}^{2}\right\|_{L^{2}\left(S_{\text {in }}\right)}^{2}+\left\|q_{w}^{1}-q_{w}^{2}\right\|_{L^{2}\left(S_{w}\right)}^{2}\right) d s,
\end{aligned}
$$

where

$$
\begin{aligned}
& \beta(t)=c_{\sigma}\left(1+\left\|\sqrt{\ell^{1}} \nabla \mathbf{u}^{1}(\cdot, t)\right\|_{L^{2}(D)}^{2}+\left\|\sqrt{\ell^{2}} \nabla \mathbf{u}^{2}(\cdot, t)\right\|_{L^{2}(D)}^{2}\right), \\
& c_{\sigma}=c(\sigma, \delta, \varepsilon, \kappa, \alpha, K),
\end{aligned}
$$

and $\sigma>0$ may be chosen small. Note that $c_{\sigma}$ depends on $\varepsilon$ even in the case that $\operatorname{div}_{h^{i}} \boldsymbol{u}^{i}$, $i=1,2$, vanish since the items $q^{i} \operatorname{div}_{h^{i}} \boldsymbol{U}$ do not disappear. Next we choose $\sigma>0$ such small enough that the integral over $|\nabla \boldsymbol{U}|^{2}+|\nabla Q|^{2}$ on the right-hand side is dominated by the corresponding integrals on the left-hand side. After neglecting some nonnegative items on the left-hand side, we arrive at Gronwall's inequality

$$
w(t) \leq \zeta(\tau, t)+\int_{\tau}^{t} \beta(s) w(s) d s
$$

with

$$
\begin{aligned}
& w(t) \stackrel{\text { def }}{=}\|\boldsymbol{U}(\cdot, t)\|_{L^{2}(D)^{2}}^{2}+\varepsilon\|Q(\cdot, t)\|_{L^{2}(D)}^{2}+\|z(\cdot, t)\|_{L^{2}(0, L)}^{2}, \\
& \zeta(\tau, t) \stackrel{\text { def }}{=} c_{\sigma} \varpi(\tau, t)\left\|h^{1}-h^{2}\right\|_{W^{1, \infty}\left(\mathcal{S}_{\tau, t}\right)}^{2}+c_{\sigma} \int_{\tau}^{t}\left(\left\|q_{\text {out }}^{1}-q_{\text {out }}^{2}\right\|_{L^{2}\left(S_{\text {out }}\right)}^{2}\right. \\
& \left.\quad+\left\|q_{\text {in }}^{1}-q_{\text {in }}^{2}\right\|_{L^{2}\left(S_{\text {in }}\right)}^{2}+\left\|q_{w}^{1}-q_{w}^{2}\right\|_{L^{2}\left(S_{W}\right)}^{2}\right) d s, \\
& \varpi(\tau, t) \stackrel{\text { def }}{=} \sum_{i=1}^{2}\left(\varepsilon\left\|q^{i}\right\|_{L^{2}\left(\tau, t ; L^{2}(D)\right)}^{2}+\left\|\boldsymbol{u}^{i}\right\|_{L^{2}\left(\tau, t ; H^{1}(D)\right)}^{2}\right)
\end{aligned}
$$

and $\beta(t)$ given by (A.3). Note that $\varpi(\tau, t)$ will be small if $0<t-\tau$ is small (see the bound in (3.1)). Since $\zeta(\tau, t)$ is non-decreasing in $t$, Gronwall's lemma and (A.4) imply the estimate

$$
w(t) \leq \zeta(\tau, t) \exp \left(\int_{\tau}^{t} \beta(s) d s\right) \text { for a.a. } t \in[\tau, T]
$$


Observe that $\int_{0}^{T} \beta(s) d s$ is bounded due to Theorem 3.1. Hence, we have

$$
w(t) \leq c \zeta(\tau, t) \quad \text { and } \quad \int_{\tau}^{t} w(s) d s \leq c(t-\tau) \zeta(\tau, t)
$$

Inserting this into the right-hand side of (A.2), we obtain the assertion (4.5) of Theorem 4.3.

It remains to prove integral inequality (A.2) from relation (A.1). Since this leads to a huge number of items, we restrict ourselves only to a few items on which we demonstrate the technique. We start with the last item on the left-hand side of (A.1) and estimate

$$
\begin{aligned}
\kappa & \int_{\tau}^{t} \int_{0}^{L}\left(\left(u_{2}^{1}-z^{1}\right)-\left(u_{2}^{2}-z^{2}\right)\right)\left(\left(u_{2}^{1} \ell^{1}-u_{2}^{2} \ell^{2}\right)-\left(z^{1}-z^{2}\right)\right) d x d s \\
= & \kappa \int_{\tau}^{t} \int_{0}^{L}\left(\left(u_{2}^{1}-u_{2}^{2}\right) U_{2}-z U_{2}-z\left(u_{2}^{1}-u_{2}^{2}\right)+|z|^{2}\right) d x d s \\
= & \kappa \int_{\tau}^{t} \int_{0}^{L}\left(\frac{1}{\ell^{1}}\left(\ell^{1} u_{2}^{1}-\ell^{1} u_{2}^{2}\right) U_{2}-z U_{2}-z\left(u_{2}^{1}-u_{2}^{2}\right)+|z|^{2}\right) d x d s \\
\geq & \kappa \int_{\tau}^{t} \int_{0}^{L}\left(\frac{1}{\ell^{1}}\left|U_{2}\right|^{2}+|z|^{2}\right) d x d s-\kappa \int_{\tau}^{t} \int_{0}^{L}\left(\frac{1}{\alpha^{2}}\left|\ell^{1}-\ell^{2}\right|\left|u_{2}^{2}\right|\left|U_{2}\right|+|z|\left|U_{2}\right|\right. \\
& \left.+\frac{1}{\alpha^{2}}|z|\left(\left|\ell^{1} u_{2}^{1}-\ell^{2} u_{2}^{2}\right|+\left|\ell^{1}-\ell^{2}\right|\left|u_{2}^{2}\right|\right)\right) d x d s \\
\geq & \kappa \int_{\tau}^{t} \int_{0}^{L}\left(\alpha^{2}\left|U_{2}\right|^{2}+|z|^{2}\right) d x d s \\
& -\kappa \int_{\tau}^{t} \int_{0}^{L}\left(1+\frac{1}{\alpha^{2}}\right)\left(|z|\left|U_{2}\right|+\left|\ell^{1}-\ell^{2}\right|\left(\left|u_{2}^{2}\right|\left|U_{2}\right|+\left|u_{2}^{2}\right||z|\right)\right) d x d s \\
\geq & \kappa \int_{\tau}^{t} \int_{0}^{L}\left(\alpha^{2}\left|U_{2}\right|^{2}+|z|^{2}\right) d x d s-\sigma \kappa \int_{\tau}^{t} \int_{0}^{L}\left|U_{2}\right|^{2} d x d s \\
& -\kappa c_{\sigma} \int_{\tau}^{t}\|z(\cdot, s)\|_{L^{2}(0, L)}^{2} d s-\kappa c_{\sigma}\left\|\ell^{1}-\ell^{2}\right\|_{L^{\infty}\left(\mathcal{S}_{\tau, t}\right)}^{2}\left\|u_{2}^{2}\right\|_{L^{2}\left(\tau, t ; L^{2}(0, L)\right)}^{2} \\
\geq & -\zeta(\tau, t)-\int_{\tau}^{t} w(s) d s
\end{aligned}
$$

for small $\sigma>0$ since $\left\|\ell^{1}-\ell^{2}\right\|_{L^{\infty}\left(\mathcal{S}_{\tau, t}\right)}^{2} \leq c(\alpha, \delta)\left\|h^{1}-h^{2}\right\|_{L^{\infty}\left(\mathcal{S}_{\tau, t}\right)}^{2}$ due to (2.10) and the embedding $\left\|u_{2}^{2}\right\|_{L^{2}\left(\tau, t ; L^{2}(0, L)\right)}^{2} \leq c\left\|\boldsymbol{u}^{2}\right\|_{L^{2}\left(\tau, t ; H^{1}(D)\right)}^{2}$.

The most extensive calculations occur in the estimation of the nonlinear items in the middle of relation (A.1). However, these are also the most interesting items in our estimation. Consider, e.g. the difference of the second and the forth item in the middle of (A.1),

$$
I:=-\frac{\rho}{2} \int_{\tau}^{t} \int_{D}\left[\left(\left(\boldsymbol{u}^{1} \cdot \nabla_{h^{1}}\right) \boldsymbol{U}\right) \cdot \mathbf{u}^{1} \ell^{1}-\left(\left(\boldsymbol{u}^{2} \cdot \nabla_{h^{2}}\right) \boldsymbol{U}\right) \cdot \mathbf{u}^{2} \ell^{2}\right] d(x, y) d s,
$$

we can decompose it into

$$
\begin{aligned}
I:= & -\frac{\rho}{2} \int_{\tau}^{t} \int_{D}\left[\left(\left(\left(\boldsymbol{u}^{1}-\boldsymbol{u}^{2}\right) \cdot \nabla_{h^{1}}\right) \boldsymbol{U}\right) \cdot \mathbf{u}^{1} \ell^{1}+\left(\left(\boldsymbol{u}^{2} \cdot\left(\nabla_{h^{1}}-\nabla_{h^{2}}\right)\right) \boldsymbol{U}\right) \cdot \mathbf{u}^{1} \ell^{1}\right. \\
& \left.+\left(\left(\boldsymbol{u}^{2} \cdot \nabla_{h^{2}}\right) \boldsymbol{U}\right) \cdot\left(\boldsymbol{u}^{1}-\boldsymbol{u}^{2}\right) \ell^{1}+\left(\left(\boldsymbol{u}^{2} \cdot \nabla_{h^{2}}\right) \boldsymbol{U}\right) \cdot \boldsymbol{u}^{2}\left(\ell^{1}-\ell^{2}\right)\right] d(x, y) d s \\
= & -I_{1}-I_{2}-I_{3}-I_{4} .
\end{aligned}
$$


We write these items on the right-hand side of (A.1) and estimate the first integral

$$
\begin{aligned}
\left|I_{1}\right|= & \mid \frac{\rho}{2} \int_{\tau}^{t} \int_{D}\left[\left(\left(u_{1}^{1}-u_{1}^{2}\right)\left(\boldsymbol{U}_{x}+m^{1} \boldsymbol{U}_{y}\right)\right.\right. \\
& \left.\left.+\left(u_{2}^{1}-u_{2}^{2}\right) \frac{1}{h^{1}-\delta} \boldsymbol{U}_{y}\right) \cdot \boldsymbol{u}^{1} \ell^{1}\right] d(x, y) d s \mid \\
= & \mid \frac{\rho}{2} \int_{\tau}^{t} \int_{D}\left[\left(\left(U_{1}+\left(\ell^{2}-\ell^{1}\right) u_{1}^{2}\right)\left(\boldsymbol{U}_{x}+m^{1} \boldsymbol{U}_{y}\right)\right.\right. \\
& \left.\left.+\left(U_{2}+\left(\ell^{2}-\ell^{1}\right) u_{2}^{2}\right) \frac{1}{h^{1}-\delta} \boldsymbol{U}_{y}\right) \cdot \mathbf{u}^{1}\right] d(x, y) d s \mid \\
\leq & c \int_{\tau}^{t} \int_{D}\left(|\boldsymbol{U}|+\left|\boldsymbol{u}^{2} \| h^{1}-h^{2}\right|\right)|\nabla \boldsymbol{U} \|| \boldsymbol{u}^{1} \mid d(x, y) d s \\
\leq & c \int_{\tau}^{t}\|\boldsymbol{U}\|_{L^{4}(D)^{2}}\|\nabla \boldsymbol{U}\|_{L^{2}(D)^{2}}\left\|\boldsymbol{u}^{2}\right\|_{L^{4}(D)^{2}} d s \\
& +c \int_{\tau}^{t}\left\|\boldsymbol{u}^{1}\right\|_{L^{4}(D)^{2}}\left\|\boldsymbol{u}^{2}\right\|_{L^{4}(D)^{2}}\|\nabla \boldsymbol{U}\|_{L^{2}(D)^{2}}\left\|h^{1}-h^{2}\right\|_{L^{\infty}\left(\mathcal{S}_{\tau, t}\right)} d s \\
\leq & c \int_{\tau}^{t}\|\boldsymbol{U}\|_{L^{4}(D)^{2}}\|\nabla \boldsymbol{U}\|_{L^{2}(D)^{2}}\left\|\boldsymbol{u}^{2}\right\|_{L^{4}(D)^{2}} d s+\sigma \int_{\tau}^{t}\|\nabla \boldsymbol{U}\|_{L^{2}(D)^{2}}^{2} d s \\
& +c_{\sigma}\left(\int_{\tau}^{t}\left\|\boldsymbol{u}^{1}\right\|_{L^{4}(D)^{2}}^{4} d s+\int_{\tau}^{t}\left\|\boldsymbol{u}^{2}\right\|_{L^{4}(D)^{2}}^{4} d s\right)\left\|h^{1}-h^{2}\right\|_{L^{\infty}\left(\mathcal{S}_{\tau, t}\right)^{2}}^{2}
\end{aligned}
$$

where $c$ is a generic constant depending on $\delta, \alpha, K$. To estimate the last item, we use the interpolation inequality (2.27) with $p=4$, which together with the first $a$ priori estimate (3.1) yields

$$
\int_{\tau}^{t}\left\|\boldsymbol{u}^{i}(\cdot, s)\right\|_{L^{4}(D)^{2}}^{4} d s \leq c\left\|\boldsymbol{u}^{i}\right\|_{L^{\infty}\left(\tau, t ; L^{2}(D)^{2}\right)}^{2}\left\|\boldsymbol{u}^{i}\right\|_{L^{\infty}\left(\tau, t ; H^{1}(D)^{2}\right)}^{2} \leq c \varpi(\tau, t)
$$

for $i=1,2$. To be concerned with the first item on the right-hand side of (A.6), we use again Hölder's inequality and the interpolation inequality (2.26) and obtain

$$
\begin{aligned}
& \|\boldsymbol{U}(\cdot, t)\|_{L^{4}(D)^{2}}\|\nabla \boldsymbol{U}(\cdot, t)\|_{L^{2}(D)^{2}}\left\|\boldsymbol{u}^{2}(\cdot, t)\right\|_{L^{4}(D)^{2}} \\
& \quad \leq \sigma\|\nabla \boldsymbol{U}\|_{L^{2}(D)^{2}}^{2}+c_{\sigma}\|\boldsymbol{U}\|_{L^{4}(D)^{2}}^{2}\left\|\boldsymbol{u}^{2}\right\|_{L^{4}(D)^{2}}^{2} \\
& \quad \leq \sigma\|\nabla \boldsymbol{U}\|_{L^{2}(D)^{2}}^{2}+c_{\sigma}\|\boldsymbol{U}\|_{L^{2}(D)^{2}}\|\nabla \boldsymbol{U}\|_{L^{2}(D)^{2}}\left\|\boldsymbol{u}^{2}\right\|_{L^{2}(D)^{2}}\left\|\nabla \boldsymbol{u}^{2}\right\|_{L^{2}(D)^{2}} \\
& \quad \leq 2 \sigma\|\nabla \boldsymbol{U}\|_{L^{2}(D)^{2}}^{2}+c_{\sigma}\|\boldsymbol{U}\|_{L^{2}(D)^{2}}^{2}\left\|\boldsymbol{u}^{2}\right\|_{L^{2}(D)^{2}}^{2}\left\|\nabla \boldsymbol{u}^{2}\right\|_{L^{2}(D)^{2}}^{2} \\
& \quad \leq 2 \sigma\|\nabla \boldsymbol{U}(\cdot, t)\|_{L^{2}(D)^{2}}^{2}+\beta(t) w(t)
\end{aligned}
$$

since $\left\|\boldsymbol{u}^{2}(\cdot, t)\right\|_{L^{2}(D)^{2}}^{2} \leq \delta^{-1}\left\|\left(\ell^{2}(t)\right)^{1 / 2} \boldsymbol{u}^{2}(\cdot, t)\right\|_{L^{2}(D)^{2}}^{2} \leq c$ is bounded for almost all $t \in[0, T]$ due to (3.3) and (3.4). Hence, we arrive at

$$
\left|I_{1}\right| \leq 3 \sigma \int_{\tau}^{t}\|\nabla \boldsymbol{U}(\cdot, s)\|_{L^{2}(D)^{2}}^{2} d s+\zeta(\tau, t)+\int_{\tau}^{t} \beta(s) w(s) d s
$$

for arbitrary small positive $\sigma$. Since the first item may be settled with the elliptic part of (A.1), $I_{1}$ is bounded by the right-hand side of Gronwall's inequality (A.4). The other 
integrals $I_{2}, I_{3}$ and $I_{4}$ in (A.5) as well as the remaining parts in (A.1) are estimated in a similar way, and we omit these calculations.

\section{Competing interests}

The authors declare that they have no competing interests.

\section{Authors' contributions}

The work presented here was carried out in collaboration between the authors. The authors contributed to every part of this study equally and read and approved the final version of the manuscript.

\section{Author details}

${ }^{1}$ Department of Mathematical Analysis and Numerical Mathematics, Faculty of Mathematics, Physics and Informatics, Comenius University, Mlynska dolina, Bratislava, 842 48, Slovakia. ${ }^{2}$ Faculty of Natural Sciences II, Institute of Mathematics, Martin Luther University Halle-Wittenberg, Halle, 06099, Germany.

\section{Acknowledgements}

We thank Mathematisches Forschungsinstitut Oberwolfach for its support and hospitality through the RiP program at Oberwolfach. Supported by the Slovak Research and Development Agency under the contract No. APVV-0134-10.

\section{Received: 6 August 2013 Accepted: 28 August 2013 Published: 18 Nov 2013}

\section{References}

1. Muha, B, Canic, S: Existence of a weak solution to a nonlinear fluid-structure interaction problem modeling the flow of an incompressible, viscous fluid in a cylinder with deformable walls. Arch. Ration. Mech. Anal. 207, 919-968 (2013)

2. Formaggia, L, Gerbeau, JF, Nobile, F, Quarteroni, A: On the coupling of the 3D and 1D Navier-Stokes equations for flow problems in compliant vessels. Comput. Methods Appl. Mech. Eng. 191, 561-582 (2001)

3. Quarteroni, A: Fluid-structure interaction for blood flow problems. In: Lecture Notes on Simulation of Fluid and Structure Interaction (AMS-AMIF Summer School). Eur. Math. Soc., Zurich (2001)

4. Quarteroni, A: Mathematical modelling of the cardiovascular system. In: Tatsien, L (ed.) Proceedings of the ICM, vol. 3, pp. 839-850. Higher Education Press, Beijing (2002)

5. Filo, J, Zaušková, A: 2D Navier-Stokes equations in a time dependent domain with Neumann type boundary conditions. J. Math. Fluid Mech. 12, 1-46 (2010)

6. Beirao da Veiga, $\mathrm{H}$ : On the existence of strong solution to a coupled fluid-structure evolution problem. J. Math. Fluid Mech. 6, 21-52 (2004)

7. Chambolle, A, Desjarins, B, Esteban, MJ, Grandmont, C: Existence of weak solutions for the unsteady interaction of a viscous fluid with an elastic plate. J. Math. Fluid Mech. 7, 368-404 (2005)

8. Desjarin, B, Esteban, MJ: On weak solutions for rigid-fluid structure interaction: compressible and incompressible models. Commun. Partial Differ. Equ. 25, 1399-1423 (2000)

9. Jin, BJ, Padula, M: In a horizontal layer with free upper surface. Commun. Pure Appl. Anal. 1, 379-415 (2002)

10. Alt, HW, Luckhaus, S: Quasilinear elliptic-parabolic differential equations. Math. Z. 183, 311-341 (1983)

11. Feistauer, M: Mathematical Methods in Fluid Dynamics. Pitman Monographs and Surveys in Pure and Applied Mathematics, vol. 67. Longman, Harlow (1993)

12. Filo, J, Luckhaus, S: Modelling surface runoff and infiltration of rain by an elliptic-parabolic equation coupled with a first-order equation on the boundary. Arch. Ration. Mech. Anal. 207, 157-182 (1999)

13. Temam, R: Navier-Stokes Equations. North-Holland, Oxford (1977)

14. Zajaczkowski, WM: Global special regular solutions to the Navier-Stokes equations in a cylindrical domain without the axis of symmetry. Topol. Methods Nonlinear Anal. 24, 69-105 (2004)

15. Evans, LC: Partial Differential Equations. Am. Math. Soc., Providence (1998)

16. Ladyzenskaja, OA, Solonnikov, VA, Uralceva, NN: Linear and Quasi-Linear Equations of Parabolic Type. Am. Math. Soc., Providence (1988)

17. Opic, B, Kufner, A: Hardy-Type Inequalities. Pitman Research Notes in Mathematics Series, vol. 219. Longman, Harlow (1990)

18. Kufner, A, John, O, Fucik, S: Function Spaces. Academia, Prague (1977)

19. Kufner, A: Weighted Sobolev Spaces. Teubner, Leipzig (1980)

10.1186/1687-2770-2013-241

Cite this article as: Filo and Pluschke: An approximation of the axially symmetric flow through a pipe-like domain with a moving part of a boundary. Boundary Value Problems 2013, 2013:241 